\title{
GROUND STATES ARE GENERICALLY A PERIODIC ORBIT
}

\author{
GONZALO CONTRERAS
}

\begin{abstract}
We prove that for an expanding transformation the maximizing measures of a generic Lipschitz function are supported on a single periodic orbit.
\end{abstract}

\section{INTRODUCTION}

Let $X$ be a compact metric space and $T: X \rightarrow X$ an expanding map. This means that $T$ is Lipschitz continuous and there are numbers $d \in \mathbb{Z}^{+}, 0<\lambda<1$ such that for every point $x \in X$ there is a neighborhood $U_{x}$ of $x$ in $X$ and continuous branches $S_{i}$, $i=1, \ldots, \ell_{x} \leq d$ of the inverse of $T$ with disjoint images $S_{i}\left(U_{x}\right)$, such that $T^{-1}\left(U_{x}\right)=$ $\bigcup_{i=1}^{\ell_{x}} S_{i}\left(U_{x}\right), T \circ S_{i}=I_{U_{x}} \forall i$, and

$$
d\left(S_{i}(y), S_{i}(z)\right) \leq \lambda d(y, z) \quad \forall y, z \in U_{x} .
$$

Given a continuous function $F: X \rightarrow \mathbb{R}$, a maximizing measure is a $T$-invariant Borel probability measure $\mu$ which maximizes the integral of $F$ among all $T$-invariant Borel probabilities:

$$
\int F d \mu=\sup \left\{\int F d \nu \mid \nu \in \mathcal{M}(T)\right\}
$$

where

$$
\mathcal{M}(T)=\{T \text {-invariant Borel probabilities in } X\} .
$$

Recall that an equilibrium state for $F$ is an invariant Borel probability $\mu_{F}$ which satisfies

$$
\mu_{F}:=\arg \max \left\{h_{\mu}(T)+\int F d \mu \mid \mu \in \mathcal{M}(T)\right\} .
$$

Ground states are the zero temperature limits of equilibrium states. This means limits of the form $\lim _{\beta \rightarrow+\infty} \mu_{\beta F}$. Here $\beta$ is interpreted as the inverse of the temperature. It is known [10, Proposition 29] that if the limit of a sequence $\left\{\mu_{\beta_{k} F}\right\}_{k}$ with $\beta_{k} \rightarrow \infty$ exists, then it has to be a maximizing measure with maximal entropy among the maximizing measures. Brémont [5] proves that the limit $\lim _{\beta \rightarrow+\infty} \mu_{\beta F}$ exists if $F$ is locally constant. Chazottes, Gambaudo and Ugalde [8] give a characterization of the limit and a new proof of Brémont's

Gonzalo Contreras was Partially supported by CONACYT, Mexico, grant 178838. 
result. Leplaideur [16] gives another proof of Brémont's theorem and a generalization: if $G$ is Hölder continuous and $F$ is locally constant, then the limit when $\beta \rightarrow \infty$ of the equilibrium states of $G+\beta F$ exists. Chazottes and Hochman [9] give an example of a Lipschitz function $F$ for which the zero temperature limit does not exist. An example with a discontinuous function was given before by Van Enter and Ruszel [21].

For generic Hölder or Lipschitz functions $F$, the maximizing measure is unique. This is proven in Contreras, Lopes, Thieullen [10] and it is presented in a general version in Jenkinson [15]. The ideas came from an analogous result for lagrangian systems by Mañé [17]. After Jenkinson lecture notes [15] the study of maximizing measures for a fixed dynamical system became known as Ergodic Optimization. Surveys of the subject are presented by Jenkinson [15] and Baraviera, Leplaideur, Lopes [1].

1.1. Theorem (Contreras, Lopes, Thieullen [10], see also Jenkinson [15]). Let $T: X \rightarrow X$ be a continuous map of a compact metric space. Let $E$ be a topological vector space which is densely and continuously embedded in $C^{0}(X, \mathbb{R})$. Write

$$
\mathcal{U}(E):=\{F \in E \mid \text { there is a unique } F \text {-maximizing measure }\} .
$$

Then $\mathcal{U}(E)$ is a countable intersection of open and dense sets.

If moreover $E$ is a Baire space, then $\mathcal{U}(E)$ is dense in $E$.

The main conjecture in Ergodic Optimization during the last decade have been wether the maximizing measure for generic Hölder or Lipschitz functions $F$ is supported on a periodic orbit. For lagrangian systems an analogous statement is known as Mañé's conjecture.

On the space $\operatorname{Lip}(X, \mathbb{R})$ of Lipschitz functions on $X$ we use the norm

$$
\|f\|:=\sup _{x \in X}|f(x)|+\sup _{x \neq y} \frac{|f(x)-f(y)|}{d(x, y)} .
$$

We denote the the first term in (1) as $\|f\|_{0}$ and the second term as $\operatorname{Lip}(f)$.

Here we prove

Theorem A. If $X$ is a compact metric space and $T: X \hookleftarrow$ is an expanding map then there is an open and dense set $\mathcal{O} \subset \operatorname{Lip}(X, \mathbb{R})$ such that for all $F \in \mathcal{O}$ there is a single $F$-maximizing measure and it is supported on a periodic orbit.

Corollary B. For an open and dense set $\mathcal{O}$ of Lipschitz functions $F$ on $X$ the zero temperature limit $\lim _{\beta \rightarrow+\infty} \mu_{\beta F}$ exists and it is supported on a single periodic orbit. 
On the negative side, for expanding transformations Bousch [3, Proposition 9, p. 306] proves that for generic continuous functions the maximizing measure is not supported on a periodic orbit. Indeed, its support is the total space [3, Rem. 7]. Bousch theorem in he case of hyperbolic sets is presented by Jenkinson in [15, Theorem 4.2].

There have been several approaches to the conjecture from which we will use some of their techniques. Write

$\mathcal{P}(E):=\{F \in E \mid$ the unique $F$-maximizing measure is supported on a periodic orbit $\}$.

Contreras, Lopes, Thieullen [10] prove that $\mathcal{P}(E)$ is open for $E=C^{\alpha}(X, \mathbb{R})$ the space of $\alpha$-Hölder continuous functions and in the $\alpha$-Hölder topology it is open and dense in $E=C^{! \alpha}(X, \mathbb{R})$, the space of functions $F: X \rightarrow \mathbb{R}$ such that

$$
\forall \eta>0 \quad \exists \varepsilon>0 \quad d(x, y)<\varepsilon \Longrightarrow|F(x)-F(y)|<\eta d(x, y)^{\alpha} .
$$

The main technique is the introduction of a sub-action $u: X \rightarrow \mathbb{R}$ to transform the function $F$ to a cohomologous function $G=F+u-u \circ T$ such that $G \leq a=\int G d \mu^{G}$, where $\mu^{G}$ is a maximizing measure for $G$ and $F$. The sub-action is defined similarly, and plays the same role, as a sub-solution of the Hamilton-Jacobi equation for Lagrangian systems. In fact analogous constructions to the weak KAM theory can be translated to this setting. In proposition 2.2 we construct a sub-action following the original method by Fathi [11] to construct weak KAM solutions. This method was used in ergodic optimization by Bousch in [2]. In fact many results from Lagrangians systems can be translated to the ergodic optimization setting, see for example Garibaldi, Lopes, Thieullen [13].

Bousch proves that $\mathcal{P}(E)$ is dense for Walters functions. Yuan and Hunt [23] prove that if a fixed measure is maximizing for an open set of functions $F$ in the Lipschitz topology, then it is supported on a periodic orbit. Their method of perturbation is the basis of the present work. Quas and Siefken [19] work in a one-sided shift. They prove that $\mathcal{P}(E)$ contains an open and dense set if $E$ is the space of super-continuous functions. They present an elegant version of the method of Yuan and Hunt. We need to modify it for Lipschitz functions and pseudo-orbits with finitely many jumps in Proposition 2.6.

Another ingredient of the proof is the following theorem. As a weak version of the conjecture, Morris [18] proves

1.2. Theorem (Morris [18]). Let $X$ be a compact metric space and $T: X \hookleftarrow$ an expanding map. There is a residual set $\mathcal{G} \subset \operatorname{Lip}(X, \mathbb{R})$ such that if $F \in \mathcal{G}$ then there is a unique $F$-maximizing measure and it has zero metric entropy. 
The idea of the proof of Theorem 1.2 is to use a periodic orbit with small action and small period constructed by Bressaud and Quas [6] and perturb $F$ so that the new minimizing measures are nearby the periodic orbit and hence have small entropy.

The original version of Theorem 1.2 is for Hölder functions in a shift of finite type. In appendix A we describe the modifications from the proof in Morris [18] needed to obtain Theorem 1.2.

In section 2 we develop the techniques from ergodic optimization that we need and present the main perturbation result in proposition 2.6. In section 3 we prove Theorem A with an argument by contradiction. We show that if the conditions for a perturbation as in proposition 2.6 do not hold then the entropy must be positive, contradicting Morris Theorem 1.2.

\section{Preliminars}

Since $X$ is compact there is a finite subcover of $\left\{U_{x}\right\}_{x \in X}$ in the definition of expanding transformation. Also there is $e_{0}>0$ such that for every $x \in X$ there is some $U_{y}$ such that the ball $B\left(x, e_{0}\right) \subset U_{y}$.

We have that $e_{0}>0$ and $0<\lambda<1$ are such that for every $x \in X$ the branches of the inverse of $T$ are well defined, injective, have disjoint images and are $\lambda$-contractions on the ball $B\left(x, e_{0}\right)$ of radius $e_{0}$ centered at $x$.

Given $F \in \operatorname{Lip}(X, \mathbb{R})$, the Lax operator for $F$ is $\mathcal{L}_{F}: \operatorname{Lip}(X, \mathbb{R}) \hookleftarrow$

$$
\mathcal{L}_{F}(u)(x)=\max _{y \in T^{-1}(x)}\{\alpha+F(y)+u(y)\}
$$

where

$$
\alpha=\alpha(F):=-\max _{\mu \in \mathcal{M}(T)} \int F d \mu .
$$

Denote the set of maximizing measures by

$$
\mathcal{M}(F):=\left\{\mu \in \mathcal{M}(T) \mid \int F d \mu=-\alpha(F)\right\}
$$

A calibrated sub-action for $F$ is a fixed point of the Lax operator $\mathcal{L}_{F}$. 


\subsection{Lemma.}

1. If $u \in \operatorname{Lip}(X, \mathbb{R})$, the Lipschitz constants satisfy

$$
\operatorname{Lip}\left(\mathcal{L}_{F}(u)\right) \leq \lambda(\operatorname{Lip}(F)+\operatorname{Lip}(u)) .
$$

In particular $\mathcal{L}_{F}(\operatorname{Lip}(X, \mathbb{R})) \subset \operatorname{Lip}(X, \mathbb{R})$.

2. If $\mathcal{L}_{F}(u)=u$, writing

$$
\bar{F}:=F+\alpha(F)+u-u \circ T
$$

we have that

(i) $\alpha(\bar{F})=-\max _{\mu \in \mathcal{M}(T)} \int \bar{F} d \mu=0$.

(ii) $\bar{F} \leq 0$.

(iii) $\mathcal{M}(F)=\mathcal{M}(\bar{F})=\{T$-invariant measures supported on $[\bar{F}=0]\}$

3. If $u \in \operatorname{Lip}(X, \mathbb{R})$ and $\beta \in \mathbb{R}$ satisfy $\mathcal{L}_{F}(u)=u+\beta$, then $\beta=0$.

\section{Proof:}

1. Given $x, y \in X$ with $d(x, y)<e_{0}$, let $\bar{y} \in T^{-1}(y)$ be such that

$$
\mathcal{L}_{F}(u)(y)=\alpha+F(\bar{y})+u(\bar{y}) .
$$

Let $S: B\left(y, e_{0}\right) \rightarrow X$ be the branch of the inverse of $T$ such that $S(y)=\bar{y}$. We have that

$$
\begin{aligned}
\mathcal{L}_{F}(u)(y)-\mathcal{L}_{F}(u)(x) & \leq \alpha+F(\bar{y})+u(\bar{y})-\alpha-F(S(x))-u(S(x)) \\
& \leq F(S(y))-F(S(x))+u(S(y))-u(S(x)) \\
& \leq \lambda(\operatorname{Lip}(F)+\operatorname{Lip}(u)) d(y, x) .
\end{aligned}
$$

The other inequality is similar.

2. Observe that for any invariant probability $\mu$ we have that

$$
\int \bar{F} d \mu=\alpha+\int F d \mu \text {. }
$$

Therefore

$$
-\alpha(\bar{F})=\max _{\mu \in \mathcal{M}(T)} \int \bar{F} d \mu=\alpha(F)+\max _{\mu \in \mathcal{M}(T)} \int F d \mu=\alpha(F)-\alpha(F)=0 .
$$

This gives (i).

(ii). Since $\mathcal{L}_{F}(u)=u$, we have that

$$
u(T(y)) \geq \alpha+F(y)+u(y) \quad \forall y \in X .
$$

Thus $\bar{F} \leq 0$. 
(iii). By the equality (4) we have that $\mathcal{M}(F)=\mathcal{M}(\bar{F})$. Since $T$ is continuous, under the weak* topology, the space $\mathcal{M}(T)$ of invariant measures is closed in the space of Borel probabilities in $X$, which is compact. Since $\bar{F}$ is continuous, the map $\mu \mapsto \int \bar{F} d \mu$ is continuous. Therefore the maximum in (i) is attained by an invariant probability.

By (ii) the function $\bar{F} \leq 0$ is non-positive. Therefore any invariant measure supported on $[\bar{F}=0]$ is maximizing for $\bar{F}$. Conversely, by (i), if $\mu$ is a maximizing measure for $\bar{F}$ then it is invariant and $\int \bar{F} d \mu=0$. Thus the support of $\mu$ must be inside $[\bar{F}=0]$.

3. Define $\bar{F}$ by (3). The hypothesis $\mathcal{L}_{F}(u)=u+\beta$ implies that $\bar{F}(y) \leq \beta$ for all $y \in X$. Therefore

$$
\beta \geq \max _{\mu \in \mathcal{M}(T)} \int \bar{F} d \mu=\alpha+\max _{\mu \in \mathcal{M}(T)} \int F d \mu=0 .
$$

The set $[\bar{F}=\beta]$ is closed and by the hypothesis $\mathcal{L}_{F}(u)=u+\beta$, it contains a whole pre-orbit. This means that there is a sequence $\left\{x_{n}\right\}_{n \in \mathbb{N}} \subset[\bar{F}=\beta]$ such that $\forall n \in \mathbb{N}, T\left(x_{n+1}\right)=x_{n}$. Let $\mu_{N}$ be the probability measure defined by

$$
\int f d \mu_{N}:=\frac{1}{N} \sum_{i=0}^{N-1} f\left(x_{i}\right), \quad \forall f \in C^{0}(X, \mathbb{R}) .
$$

Since $X$ is compact, the space of Borel probability measures on $X$ is compact. Therefore there is a convergent subsequence $\lim _{k} \mu_{N_{k}}=\nu$. The probability $\nu$ is supported on $[\bar{F}=\beta]$ and it is $T$-invariant. We have that

$$
\beta=\int \bar{F} d \nu=\alpha+\int F d \nu \leq 0 .
$$

For $f: X \rightarrow \mathbb{R}$ continuous, write

$$
\|f\|_{0}:=\sup _{x \in X}|f(x)|
$$

2.2. Proposition. There exists a Lipschitz calibrated sub-action.

Proof: By (2), the Lax operator $\mathcal{L}_{F}$ leaves invariant the space

$$
\mathbb{E}:=\left\{u \in \operatorname{Lip}(X, \mathbb{R}) \mid \operatorname{Lip}(u) \leq \frac{\lambda \operatorname{Lip}(F)}{1-\lambda}\right\} .
$$


Fix $x_{0} \in X$. Arzelà-Ascoli Theorem implies that the quotient space $\mathbb{E} / \mathbb{R}:=\mathbb{E} /\{$ constants $\}$ with the supremum norm $\|f+\mathbb{R}\|_{\mathbb{E} / \mathbb{R}}:=\sup _{x \in X}\left|f(x)-f\left(x_{0}\right)\right| \leq 2\|f\|_{0}$ is compact.

If $a \in \mathbb{R}$ then $\mathcal{L}_{F}(u+a)=\mathcal{L}_{F}(u)+a$. Therefore $\mathcal{L}_{F}: \mathbb{E} / \mathbb{R} \rightarrow \mathbb{E} / \mathbb{R}$ is well defined. If $u, v \in \mathbb{E}, x \in X$ and $x_{u}^{*} \in X$ is such that $\mathcal{L}(u)=\alpha+F\left(x_{u}^{*}\right)+u\left(x_{u}^{*}\right)$, then

$$
\begin{aligned}
\mathcal{L}_{F}(u)(x)-\mathcal{L}_{F}(v)(x) & \leq \alpha+F\left(x_{u}^{*}\right)+u\left(x_{u}^{*}\right)-\alpha-F\left(x_{u}^{*}\right)-v\left(x_{u}^{*}\right) \\
& \leq u\left(x_{u}^{*}\right)-v\left(x_{u}^{*}\right) \leq\|u-v\|_{0} . \\
\left\|\mathcal{L}_{F}(u)-\mathcal{L}_{F}(v)\right\|_{0} & \leq\|u-v\|_{0} . \\
\left\|\left(\mathcal{L}_{F}(u)+\mathbb{R}\right)-\left(\mathcal{L}_{F}(v)+\mathbb{R}\right)\right\|_{\mathbb{E} / \mathbb{R}} & \leq 2\|u-v\|_{0} .
\end{aligned}
$$

Choosing representatives for $u+\mathbb{R}$ and $v+\mathbb{R}$ such that $u\left(x_{0}\right)=v\left(x_{0}\right)$, we have that $\|u-v\|_{0}=\|(u+\mathbb{R})-(v+\mathbb{R})\|_{\mathbb{E}}$. Thus

$$
\left\|\mathcal{L}_{F}(u+\mathbb{R})-\mathcal{L}_{F}(v+\mathbb{R})\right\|_{\mathbb{E}} \leq 2\|(u+\mathbb{R})-(v+\mathbb{R})\|_{\mathbb{E}} .
$$

Therefore the space $\mathbb{E} / \mathbb{R}$ is compact and convex and on it $\mathcal{L}_{F}$ is continuous. By Schauder Theorem [14, Theorem 18.10 , p. 197] $\mathcal{L}_{F}$ has a fixed point in $\mathbb{E} / \mathbb{R}$. In fact $\mathcal{L}_{F}$ is nonexpanding in the supremum norm and a simpler fixed point applies ${ }^{1}$ [14, Theorem 3.1, p. 28].

Then there is $u \in \mathbb{E}$ and $\beta \in \mathbb{R}$ such that $\mathcal{L}_{F}(u)=u+\beta$. By Lemma 2.1-3, we have that $\beta=0$.

If $u$ is a calibrated sub-action, every point $z \in X$ has a calibrating pre-orbit, $\left(z_{k}\right)_{k \leq 0}$ such that $T\left(z_{-k}\right)=z_{-k+1}, T^{i}\left(z_{-i}\right)=z_{0}=z$ and

$$
u\left(z_{k+1}\right)=u\left(z_{k}\right)+\alpha+F\left(z_{k}\right), \quad \forall k \leq-1 .
$$

Or equivalently, since $T\left(z_{k}\right)=z_{k+1}$,

$$
\bar{F}\left(z_{k}\right)=0, \quad \forall k \leq-1 .
$$

The iteration of equality (5) gives

$$
\forall k \leq-1, \quad u\left(z_{0}\right)=u\left(z_{-k}\right)+k \alpha+\sum_{i=-k}^{-1} F\left(z_{i}\right)
$$

for any calibrating pre-orbit.

\footnotetext{
${ }^{1}$ Let $\mathbb{F}=\mathbb{E} / \mathbb{R}$ with the norm $\|u+\mathbb{R}\|_{\mathbb{F}}:=\min _{a \in \mathbb{R}}\|u+a\|_{0}$. Then $\left(\mathbb{F},\|\cdot\|_{\mathbb{F}}\right)$ is compact, convex and $\mathcal{L}_{F}$ has Lipschitz constant 1 on $\|\cdot\|_{\mathbb{F}}$.
} 
2.3. Lemma. If there is a periodic orbit $\mathcal{O}(y)$ such that for any calibrated sub-action the $\alpha$-limit of every calibrating pre-orbit is $\mathcal{O}(y)$ then every maximizing measure has support on $\mathcal{O}(y)$.

Proof: It is enough to prove the following

Claim: If $\nu$ is an ergodic maximizing measure there is a Borel set $Y$ with $\nu(Y)=1$ such that for any $y \in Y$ there is a calibrating pre-orbit $\left\{x_{n}\right\}_{n \in \mathbb{N}}$ of a calibrated sub-action $u$ such that $y \in \alpha-\lim \left\{x_{n}\right\}_{n \in \mathbb{N}}$.

We will prove it by applying Poincaré Recurrence Theorem to the inverse of the natural (bijective) extension of $T$.

There is a canonical way of embedding an expanding map into an invertible map as follows. Let $\mathbb{X} \subset X^{\mathbb{N}}=\prod_{n \in \mathbb{N}} X$ be the space of sequences $\left\{x_{n}\right\}_{n \in \mathbb{N}}$ with $T\left(x_{n+1}\right)=x_{n}$ for every $n \in \mathbb{N}$, endowed with the subspace topology induced by the product tolopogy on $X^{\mathbb{N}}$. Since $X$ is compact, by Tychonof Theorem $X^{\mathbb{N}}$ is compact and then, as a closed subspace, $\mathbb{X}$ is compact. Let $\mathbb{T}: \mathbb{X} \rightarrow \mathbb{X}$ be defined by $\mathbb{T}\left(\left\{x_{n}\right\}_{n \in \mathbb{N}}\right)=\left\{T\left(x_{n}\right)\right\}_{n \in \mathbb{N}}=\left\{\ldots, x_{1}, x_{0}, T\left(x_{0}\right)\right\}$. Then $\mathbb{T}$ is a homeomorphism with inverse $\mathbb{T}^{-1}\left(\left\{x_{n}\right\}_{n \in \mathbb{N}}\right)=\left\{x_{n+1}\right\}_{n \in \mathbb{N}}$. We have the semiconjugacy

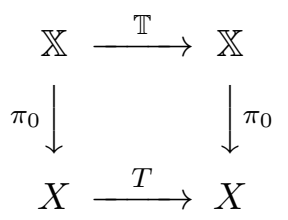

given by $\pi_{0}\left(\left\{x_{n}\right\}_{n \in \mathbb{N}}\right)=x_{0}$. The projection $\pi_{0}$ is continuous and hence Borel measurable. There is a natural way of lifting invariant measures as follows (cf. Bowen [4, §1.C]). If $f \in C^{0}(\mathbb{X}, \mathbb{R})$ define $f^{*} \in C^{0}(X, \mathbb{R})$ by

$$
f^{*}(x)=\min f\left(\pi_{0}^{-1}\{x\}\right) .
$$

If $\mu$ is a $T$-invariant Borel probability on $X$ define $\tilde{\mu}$ on $\operatorname{Borel}(\mathbb{X})$ by

$$
\tilde{\mu}(f):=\lim _{n} \mu\left(\left(f \circ \mathbb{T}^{n}\right)^{*}\right), \quad \forall f \in C^{0}(\mathbb{X}, \mathbb{R}) .
$$

Then $\tilde{\mu}$ is $\mathbb{T}$-invariant and $\left(\pi_{0}\right)_{*}(\tilde{\mu})=\mu$.

Suppose that $\nu$ is an ergodic maximizing measure for $F \in \operatorname{Lip}(X, \mathbb{R})$ and let $\tilde{\nu}$ be its invariant lift to $\mathbb{X}$ as defined above. The measure $\tilde{\nu}$ is $\mathbb{T}$-invariant and thus also $\mathbb{T}^{-1}$ invariant. Then $\operatorname{supp}(\tilde{\nu})$ is $\mathbb{T}^{-1}$-invariant. Let $\mathbb{Y}$ be the set of $\mathbb{T}^{-1}$-recurrent points in $\operatorname{supp}(\tilde{\nu})$ and $Y:=\pi_{0}(\mathbb{Y})$. Then $\nu(Y)=\tilde{\nu}\left(\pi_{0}^{-1}(Y)\right) \geq \tilde{\nu}(\mathbb{Y})=1$. If $y \in Y$ then there 
is $\tilde{y} \in \pi_{0}^{-1}(y) \in \mathbb{Y}$ such that $\tilde{y}$ is $\mathbb{T}^{-1}$-recurrent, i.e. $\tilde{y} \in \omega$-lim $\left(\tilde{y}, \mathbb{T}^{-1}\right)$. We have that $\tilde{y}=\left\{y_{n}\right\}_{n \in \mathbb{N}}$ is a pre-orbit of $T$ in $\operatorname{supp}(\nu)$ with $y_{0}=y$ and $y \in \alpha-\lim \left(\left\{y_{n}\right\}_{n \in \mathbb{N}}\right)$.

Let $u$ be any calibrated sub-action. Let $\bar{F}$ be defined by (3). By Lemma 2.1-2.(iii) we have that $\left\{y_{n}\right\}_{n \in \mathbb{N}} \subset \operatorname{supp}(\nu) \subset[\bar{F}=0]$. Thus by the remark in (6) the pre-orbit $\left\{y_{n}\right\}_{n \in \mathbb{N}}$ calibrates $u$.

We say that a sequence $\left(x_{n}\right)_{n \in \mathbb{N}} \subset X$ is a $\delta$-pseudo-orbit if $d\left(x_{n+1}, T\left(x_{n}\right)\right) \leq \delta, \forall n \in \mathbb{N}$.

We say that the orbit of $y \varepsilon$-shadows a pseudo-orbit $\left(x_{n}\right)_{n \in \mathbb{N}}$ if $\forall n \in \mathbb{N}, d\left(T^{n}(y), x_{n}\right)<\varepsilon$.

\subsection{Proposition (Shadowing Lemma).}

If $\left(x_{k}\right)_{k \in \mathbb{N}}$ is a $\delta$-pseudo-orbit with $\delta<(1-\lambda) e_{0}$ then there is $y \in X$ whose orbit $\varepsilon$-shadows $\left(x_{k}\right)_{k \in \mathbb{N}}$ with $\varepsilon=\frac{\delta}{1-\lambda}$. If $\left(x_{k}\right)_{k \in \mathbb{N}}$ is a periodic pseudo-orbit then $y$ is a periodic orbit with the same period.

Proof: Write $B(x, r):=\{z \in X \mid d(z, x) \leq r\}$ and $a:=\frac{\lambda \delta}{1-\lambda}$. Let $S_{k}$ be the branch of the inverse of $T$ such that $S_{k}\left(T\left(x_{k}\right)\right)=x_{k}$. Since $a+\delta<e_{0}$, we have that

$$
S_{k}\left(B\left(x_{k+1}, a\right)\right) \subseteq S_{k}\left(B\left(T\left(x_{k}\right), a+\delta\right)\right) \subseteq B\left(x_{k}, \lambda(a+\delta)\right)=B\left(x_{k}, a\right) .
$$

Let $y \in X$ be given by

$$
y \in \bigcap_{k=0}^{\infty} S_{0} \circ \cdots \circ S_{k}\left(B\left(x_{k+1}, a\right)\right) .
$$

The point $y$ exists and is unique because it is the intersection of a nested family of nonempty compact sets with diameter smaller than $2 a \lambda^{k}$. We have that $T^{k}(y) \in B\left(x_{k}, a\right)$. Thus $y a$-shadows $\left(x_{k}\right)$. Now suppose $\left(x_{k}\right)$ is $p$-periodic. Then also $T^{p}(y) a$-shadows $\left(x_{k}\right)$. The uniqueness of $y$ implies that $T^{p}(y)=y$.

\subsection{Corollary.}

If $T^{p}(y)=y$ and $\left(z_{k}\right)_{k \leq 0}$ is a pre-orbit which $(1-\lambda) e_{0}$-shadows the orbit $\mathcal{O}(y)$ of $y$, i.e. $\forall k \leq 0, T\left(z_{k}\right)=z_{k+1}$ and $d\left(z_{k}, T^{k \bmod p}(y)\right)<(1-\lambda) e_{0}$, then the $\alpha$-limit of $\left(z_{k}\right)$ is $\mathcal{O}(y)$. Proof: Let $w \in \alpha-\lim \left(z_{k}\right)$. Then there is a sequence $k_{n} \rightarrow-\infty$ such that $\lim _{n} z_{k_{n}}=$ $w$. Extracting a subsequence if necessary, we may assume that $k_{n}(\bmod p)$ is constant. Then there is $\ell \in \mathbb{Z}_{p}$ such that $d\left(z_{k_{n}}, T^{\ell}(y)\right)<(1-\lambda) e_{0}$ for all $n$. The argument in Proposition 2.4 shows that $d\left(z_{k_{n}}, T^{\ell}(y)\right)<\lambda^{k_{n}} e_{0}$. Therefore $w=\lim _{n} z_{k_{n}}=T^{\ell}(y) \in$ $\mathcal{O}(y)$. It follows that $\alpha-\lim \left(z_{k}\right)=\mathcal{O}(y)$. 
We show now a condition which allows to obtain a perturbation with maximizing measure supported on a periodic orbit. The argument appeared first in Yuan and Hunt [23]. The proof below is a modification that we shall need of the arguments by Quas and Siefken [19] which we adapt to pseudo-orbits.

Let $y \in \operatorname{Per}(T)=\cup_{p \in \mathbb{N}^{+}} \operatorname{Fix}\left(T^{p}\right)$ be a periodic point for $T$. Let $P_{y}$ be the set of Lipschitz functions $F \in \operatorname{Lip}(X, \mathbb{R})$ such that there is a unique $F$-maximizing measure and it is supported on the positive orbit of $y$. Let $\mathcal{U}_{y}$ be the interior of $P_{y}$ in $\operatorname{Lip}(X, \mathbb{R})$.

2.6. Proposition. Let $F, u \in \operatorname{Lip}(X, \mathbb{R})$ with $\mathcal{L}_{F}(u)=u$ and let $\bar{F}$ be defined by (3).

Suppose that there exists $M \in \mathbb{N}^{+}$such that for every $Q>1$ and $\delta_{0}>0$ there exist $0<\delta<\delta_{0}$ and a $p(\delta)$-periodic $\delta$-pseudo-orbit $\left(x_{k}^{\delta}\right)_{k}$ in $[\bar{F}=0]$ with at most $M$ jumps such that $\frac{\gamma_{\delta}}{\delta} \geq Q$, where $\gamma_{\delta}:=\min _{0 \leq i<j<p(\delta)} d\left(x_{i}^{\delta}, x_{j}^{\delta}\right)$.

Then $F$ is in the closure of $\cup_{y \text { periodic }} \mathcal{U}_{y}$.

Proof: Observe that fixing $u \in \operatorname{Lip}(X, \mathbb{R})$, for any $H \in \operatorname{Lip}(X, \mathbb{R})$ the functions $H$ and $H+\alpha(F)+u-u \circ T$ have the same maximizing measures. Therefore it is enough to prove that the function $\bar{F}$ is in the closure of $\cup_{y \text { periodic }} \mathcal{U}_{y}$.

Let $\varepsilon>0$. We will show a perturbation of $F$ with Lipschitz norm smaller than $\varepsilon$ such that it has a unique maximizing measure supported on a periodic orbit. Moreover, we will exhibit a neighborhood of the perturbed function in which the same periodic orbit is the unique maximizing measure for all functions in the neighborhood. The neighborhood will depend on the periodic orbit.

Let

$$
\begin{aligned}
K & :=\max \left\{\frac{M \operatorname{Lip}(\bar{F})}{(1-\lambda)^{2}}, \frac{\operatorname{Lip}(\bar{F})+2}{1-\lambda}\right\}, \\
\rho & :=\frac{3 K \delta}{\varepsilon} \\
\gamma_{3} & :=\frac{1}{\operatorname{Lip}(T)}\left(\gamma_{\delta}-\frac{2 \delta}{1-\lambda}\right)-\lambda \rho .
\end{aligned}
$$

Assume that $\delta, \gamma_{\delta}$ and $\frac{\delta}{\gamma_{\delta}}$ are so small that $\delta, \rho, \gamma_{3}$ are all positive, smaller than $(1-\lambda) e_{0}$ and that

$$
\begin{aligned}
2 K \delta-\varepsilon \rho & =:-2 b<0 . \\
2 K \delta+K \rho-\varepsilon \gamma_{3} & =:-2 a<0 .
\end{aligned}
$$

Let $y$ be the $p$-periodic point which $\left(\frac{\delta}{1-\lambda}\right)$-shadows $\left(x_{k}\right)$. Write $y_{k}:=T^{k}(y)$ and

$$
\mathcal{O}(y)=\left\{T^{i}(y) \mid i=0, \ldots, p-1\right\}=\left\{y_{0}, \ldots, y_{p-1}\right\} .
$$


For a function $G: X \rightarrow \mathbb{R}$ write

$$
\langle G\rangle(y)=\frac{1}{p} \sum_{i=0}^{p-1} G\left(T^{i}(y)\right) .
$$

Let $n_{i}, i=1, \ldots, \ell, \ell \leq M$, be the jumps of $\left(x_{k}\right)$; i.e. $d\left(T\left(x_{k}\right), x_{k+1}\right)=0$ if $k \in$ $\{0, \ldots, p-1\} \backslash\left\{n_{1}, \ldots, n_{\ell}\right\}$. Using Proposition 2.4, we have that

$$
\begin{aligned}
\left|\sum_{k=1+n_{i-1}}^{n_{i}} \bar{F}\left(y_{k}\right)-\sum_{k=1+n_{i-1}}^{n_{i}} \bar{F}\left(x_{k}\right)\right| & \leq \sum_{k=1+n_{i-1}}^{n_{i}} \operatorname{Lip}(\bar{F}) d\left(y_{k}, x_{k}\right) \leq \sum_{k=1}^{n_{i}-n_{i-1}} \lambda^{k-1} \frac{\delta}{1-\lambda} \operatorname{Lip}(\bar{F}) \\
& \leq \frac{\operatorname{Lip}(\bar{F})}{(1-\lambda)^{2}} \delta
\end{aligned}
$$

Thus

$$
\left|\sum_{k=0}^{p-1} \bar{F}\left(y_{k}\right)-\sum_{k=0}^{p-1} \bar{F}\left(x_{k}\right)\right| \leq \frac{M \operatorname{Lip}(\bar{F})}{(1-\lambda)^{2}} \delta .
$$

By hypothesis $\forall k, \bar{F}\left(x_{k}\right)=0$, thus $\sum_{0}^{p-1} \bar{F}\left(x_{k}\right)=0$. Therefore

$$
\begin{gathered}
\sum_{k=0}^{p-1} \bar{F}\left(y_{k}\right) \geq-\frac{M \operatorname{Lip}(\bar{F})}{(1-\lambda)^{2}} \delta \geq-K \delta \\
\langle\bar{F}\rangle(y) \geq-\frac{K \delta}{p} .
\end{gathered}
$$

Observe that if $0 \leq i<j<p$,

$$
d\left(y_{i}, y_{j}\right) \geq-d\left(y_{i}, x_{i}\right)+d\left(x_{i}, x_{j}\right)-d\left(x_{j}, y_{j}\right) \geq \gamma_{\delta}-\frac{2 \delta}{1-\lambda}=: \gamma_{2} .
$$

Claim: Assume that $d\left(z, y_{k}\right) \leq \rho \ll e_{0}$. Take $w_{1} \in T^{-1}\{z\}$ such that $d\left(w_{1}, y_{k-1}\right)<\lambda \rho$. If $w_{2} \in T^{-1}\{z\} \backslash\left\{w_{1}\right\}$ then

$$
d\left(w_{2}, \mathcal{O}(y)\right) \geq \gamma_{3}:=\frac{\gamma_{2}}{\operatorname{Lip}(T)}-\lambda \rho \gg \delta .
$$

Proof: Let $y_{j} \in \mathcal{O}(y)$ be such that $d\left(w_{2}, \mathcal{O}(y)\right)=d\left(w_{2}, y_{j}\right)$.

Let $S$ be the branch of the inverse of $T$ such that $S(z)=w_{1}$. If $x, y \in B\left(z, e_{0}\right)$ then

$$
d(S(x), S(y)) \geq \operatorname{Lip}(T)^{-1} d(T(S(x)), T(S(y)))=\operatorname{Lip}(T)^{-1} d(x, y) .
$$

This implies that $\lambda \geq \operatorname{Lip}(T)^{-1}$. We also get that $B\left(w_{1},(\operatorname{Lip} T)^{-1} e_{0}\right) \subset S\left(B\left(z, e_{0}\right)\right)$ and then $T$ is injective in the ball $B\left(w_{1}, \operatorname{Lip}(T)^{-1} e_{0}\right)$. In particular $d\left(w_{2}, w_{1}\right) \geq \operatorname{Lip}(T)^{-1} e_{0}$. 
If $j=k-1$ then

$$
\begin{aligned}
d\left(w_{2}, \mathcal{O}(y)\right) & =d\left(w_{2}, y_{j}\right)=d\left(w_{2}, y_{k-1}\right) \geq d\left(w_{2}, w_{1}\right)-d\left(w_{1}, y_{k-1}\right) \\
& \geq \operatorname{Lip}(T)^{-1} e_{0}-\lambda \rho>\gamma_{3} .
\end{aligned}
$$

If $j \neq k-1$ then

$$
\begin{gathered}
\gamma_{2} \leq d\left(y_{k}, y_{j+1}\right) \leq d\left(y_{k}, z\right)+d\left(z, y_{j+1}\right) \leq \rho+\operatorname{Lip}(T) d\left(w_{2}, y_{j}\right) . \\
d\left(w_{2}, y_{j}\right) \geq \frac{\gamma_{2}}{\operatorname{Lip}(T)}-\frac{\rho}{\operatorname{Lip}(T)} \geq \frac{\gamma_{2}}{\operatorname{Lip}(T)}-\lambda \rho .
\end{gathered}
$$

This proves the claim.

Now we make two perturbations to $\bar{F}$. The first perturbation is the addition of $-\varepsilon g(x)$, where

$$
g(x):=d(x, \mathcal{O}(y))
$$

This is a perturbation with

$$
\|\varepsilon g\|_{0} \leq \varepsilon \operatorname{diam} X, \quad \operatorname{Lip}(\varepsilon g)=\varepsilon .
$$

The second is a perturbation by any function with

$$
\|h\|_{0}<\frac{K \delta}{2 p}, \quad \operatorname{Lip}(h) \leq 1
$$

This perturbation depends on $\mathcal{O}(y)$, and in particular on its period $p$. We shall prove that the function $G_{1}:=\bar{F}-\varepsilon g+h$ has a unique maximizing measure supported on the periodic orbit $\mathcal{O}(y)$. Since the set of such functions $G_{1}$ contains an open ball centered at $\bar{F}-\varepsilon g$, this proves the proposition.

Let

$$
G=\bar{F}-\varepsilon g+h+\beta=G_{1}+\beta,
$$

where

$$
\beta=-\sup _{\mu \in \mathcal{M}(T)} \int(\bar{F}-\varepsilon g+h) d \mu
$$

It is enough to prove the claim for $G$ because $G$ and $G_{1}$ have the same maximizing measures.

Using (10), we have that

$$
\begin{aligned}
\beta & \leq-\langle\bar{F}-\varepsilon g+h\rangle(y)=-\langle\bar{F}+h\rangle(y) \\
& \leq-\langle\bar{F}\rangle(y)+\|h\|_{0} \\
& \leq \frac{K \delta}{p}+\|h\|_{0}
\end{aligned}
$$


Let $v$ be a calibrated sub-action for $G, \mathcal{L}_{G}(v)=v$. Given any $z \in X$ let $\left(z_{k}\right)_{k \leq 0}$ be a pre-orbit of $z$ which calibrates $v$. Let $0>t_{1}>t_{2}>\cdots$ be the times on which $d\left(z_{k}, \mathcal{O}(y)\right)>\rho$. If $t_{n+1}<t_{n}-1$ there is $s_{n} \in \mathbb{Z}$ such that the orbit segment $\left(z_{k}\right)_{k=t_{n+1}+1}^{t_{n}-1}$ $\rho$-shadows $\left(y_{-i+s_{n}}\right)_{i=t_{n}-t_{n+1}-1}^{1}$, thus

$$
d\left(z_{-i+t_{n}}, y_{-i+s_{n}}\right) \leq \lambda^{i-1} \rho, \quad \forall n \in \mathbb{N}, \quad \forall i=1, \ldots, t_{n}-t_{n+1}-1 .
$$

By the Claim, we have that

$$
t_{n+1}<t_{n}-1 \quad \Longrightarrow \quad d\left(z_{t_{n+1}}, \mathcal{O}(y)\right) \geq \gamma_{3} .
$$

Since both terms in $\bar{F}-\varepsilon g$ are non-positive, from (12) and (14) we obtain

$$
G \leq h+\beta \leq \frac{K \delta}{p}+2\|h\|_{0} .
$$

On a shadowing segment we have

$$
\left|\sum_{t_{n+1}+1}^{t_{n}-1} G\left(z_{k}\right)-\sum_{s_{n}-t_{n}+t_{n+1}+1}^{s_{n}-1} G\left(y_{k}\right)\right| \leq \operatorname{Lip}(G) \sum_{i=0}^{+\infty} \lambda^{i} \rho \leq \operatorname{Lip}(G) \frac{\rho}{1-\lambda} \leq K \rho .
$$

Write

$$
t_{n}-t_{n+1}-1=m p+r
$$

with $0 \leq r<p$ and separate the shadowing segment in $m$ loops along the orbit $\mathcal{O}(y)$ and a residue with at most $p-1$ iterates. Using (16) for $(p-1)$ times and (17), we have that

$$
\sum_{t_{n+1}+1}^{t_{n}-1} G\left(z_{k}\right) \leq m p\langle G\rangle(y)+(p-1) \frac{K \delta}{p}+2(p-1)\|h\|_{0}+\operatorname{Lip}(G) \frac{\rho}{1-\lambda} .
$$

By the definition of $\beta$ we have that $\langle G\rangle(y) \leq 0$. Therefore

$$
\sum_{t_{n+1}+1}^{t_{n}-1} G\left(z_{k}\right) \leq(p-1) \frac{K \delta}{p}+2(p-1)\|h\|_{0}+K \rho .
$$

On the points $z_{t_{n}}$ we have that $d\left(z_{t_{n}}, \mathcal{O}(y)\right)>\rho$. Using (12), (16), (11) and (8),

$$
G\left(z_{t_{n}}\right) \leq \bar{F}\left(z_{t_{n}}\right)-\varepsilon \rho+\|h+\beta\|_{0} \leq 0-\varepsilon \rho+\frac{K \delta}{p}+2\|h\|_{0}<-b<0 .
$$

In particular, this holds when $t_{n}=t_{n-1}-1$.

When $t_{n+1}<t_{n}-1$, using (12), (15) and (16), we have that

$$
G\left(z_{t_{n+1}}\right) \leq 0-\varepsilon \gamma_{3}+\|h+\beta\|_{0} \leq-\varepsilon \gamma_{3}+\frac{K \delta}{p}+2\|h\|_{0} .
$$


Thus, adding (18) and (20), and using (11) and (9),

$$
t_{n+1}<t_{n}-1 \quad \Longrightarrow \quad \sum_{t_{n+1}}^{t_{n}-1} G\left(z_{k}\right) \leq 2 p\|h\|_{0}+K \delta+K \rho-\varepsilon \gamma_{3}<-a<0 .
$$

From (13) we have that $\alpha(G)=0$. Since by definition $\left(z_{k}\right)_{k \leq 0}$ is a calibrating pre-orbit for $v$, as in (7), we have that for all $k<0$,

$$
v(z)=v\left(z_{k}\right)+\sum_{i=k+1}^{-1} G\left(z_{i}\right)
$$

Since $v$ is finite, we get that

$$
\sum_{-\infty}^{-1} G\left(z_{k}\right) \geq-2\|v\|_{0}>-\infty
$$

From (19) and (21) we obtain that the sequence $t_{n}$ is finite. Since $\rho<(1-\lambda) e_{0}$, from Corollary 2.5 we get that every calibrating pre-orbit has $\alpha$-limit $\mathcal{O}(y)$. By Lemma 2.3, this implies that every maximizing measure for $G$ has support on $\mathcal{O}(y)$.

\section{Proof of Theorem A}

\section{Proof of theorem A:}

We prove that $\mathcal{O}:=\bigcup_{y \in \operatorname{Per}(T)} \mathcal{U}_{y}$ is open and dense. It is clearly open.

Suppose, by contradiction, that there is a non-empty open set

$$
\mathcal{W} \subset \operatorname{Lip}(X, \mathbb{R})
$$

which is disjoint from $\bigcup_{y \in \operatorname{Per}(T)} \mathcal{U}_{y}$. By Theorem 1.2 and Remark A.3 we can choose $F \in \mathcal{W}$ such that it has an ergodic maximizing measure $\mu$ with entropy

$$
h_{\mu}(T)=0 .
$$

By Lemma 2.1-2.(iii) for any calibrating subaction $u$ for $F$, we have that $\operatorname{supp}(\mu) \subset[\bar{F}=0]$, where $\bar{F}$ is from (3). Let $q \in \operatorname{supp}(\mu) \subset[\bar{F}=0]$ be a generic point for $\mu$, i.e. for any continuous function $f: X \rightarrow \mathbb{R}$,

$$
\int f d \mu=\langle f\rangle(q)=\lim _{N} \frac{1}{N} \sum_{i=0}^{N-1} f\left(T^{i}(q)\right) .
$$

Since $F$ is not in the closure of $\bigcup_{y \in \operatorname{Per}(T)} \mathcal{U}_{y}$, by Proposition 2.6 with $M=2$, we have the following 


\subsection{Statement.}

There is $Q>1$ and $\delta_{0}>0$ such that if $0<\delta<\delta_{0}$ and $\left(x_{k}\right)_{k \geq 0} \subset \mathcal{O}(q)$ is a p-periodic $\delta$-pseudo-orbit with at most 2 jumps made with elements of the positive orbit of $q$ then $\gamma=\min _{1 \leq i<j<p} d\left(x_{i}, x_{j}\right)<\frac{1}{2} Q \delta$.

Let $N_{0}$ be such that

$$
2 Q^{-N_{0}}<\delta_{0}
$$

Fix a point $w \in \operatorname{supp}(\mu)$ for which Brin-Katok Theorem holds [7], i.e.

$$
h_{\mu}(T)=-\lim _{L \rightarrow+\infty} \frac{1}{L} \log \mu(V(w, L, \varepsilon)),
$$

where $V(w, L, \varepsilon)$ is the dynamic ball:

$$
V(w, L, \varepsilon):=\left\{x \in X \mid d\left(T^{k} x, T^{k} w\right)<\varepsilon, \forall k=0, \ldots, L\right\} .
$$

Given $N>N_{0}$ let $0 \leq t_{1}^{N}<t_{2}^{N}<\cdots$ be all the $\frac{1}{2} Q^{-N}$ returns to $w$, i.e.

$$
\left\{t_{1}^{N}, t_{2}^{N}, \ldots\right\}=\left\{n \in \mathbb{N} \mid d\left(T^{n} q, w\right) \leq \frac{1}{2} Q^{-N}\right\} .
$$

We need the following

3.2. Proposition. For any $\ell \geq 0, \quad t_{\ell+1}^{N}-t_{\ell}^{N} \geq \sqrt{2}^{N-N_{0}-1}$.

Using Proposition 3.2 we continue the proof of Theorem A.

Write

$$
B(w, r):=\{x \in X \mid d(x, w) \leq r\} .
$$

Given $N \gg N_{0}$, let $f_{N}: X \rightarrow \mathbb{R}$ be a continuous function such that $0 \leq f \leq 1$, $\left.f\right|_{B\left(w, \frac{1}{2} Q^{-N-1}\right)} \equiv 1$ and $\operatorname{supp}(f) \subseteq B\left(w, \frac{1}{2} Q^{-N}\right)$. Using that $q$ is a generic point for $\mu$ and Proposition 3.2, we have that

$$
\begin{aligned}
\mu\left(B\left(w, \frac{1}{2} Q^{-N-1}\right)\right) & \leq \int f_{N} d \mu=\lim _{L \rightarrow+\infty} \frac{1}{L} \sum_{i=0}^{L-1} f_{N}\left(T^{i} q\right) \\
& \leq \lim _{L \rightarrow+\infty} \frac{1}{L} \#\left\{0 \leq i<L \mid d\left(T^{i} q, w\right) \leq \frac{1}{2} Q^{-N}\right\} \\
& \leq \lim _{L \rightarrow+\infty} \frac{1}{L} \#\left\{\ell \mid t_{\ell}^{N} \leq L\right\} \\
& \leq \sqrt{2}^{-N+N_{0}+1} .
\end{aligned}
$$


Recall that the dynamic ball about $w$ is

$$
V(w, L, \varepsilon):=\left\{x \in X \mid d\left(T^{k} x, T^{k} w\right)<\varepsilon, \forall k=0, \ldots, L\right\} .
$$

We have that

$$
V(w, L, \varepsilon)=S_{1} \circ \cdots \circ S_{L}\left(B\left(T^{L} w, \varepsilon\right)\right),
$$

where $S_{k}$ is the branch of the inverse of $T$ such that $S_{k}\left(T^{k} w\right)=T^{k-1} w$. Therefore

$$
V(w, L, \varepsilon) \subseteq B\left(w, \lambda^{L} \varepsilon\right) .
$$

Let $N$ be such that

$$
\frac{1}{2} Q^{-N-2} \leq \lambda^{L} \varepsilon \leq \frac{1}{2} Q^{-N-1}
$$

Then

$$
-N \leq L \frac{\log \lambda}{\log Q}+\frac{\log (2 \varepsilon)}{\log Q}+2
$$

Using (29), we have that

$$
\begin{aligned}
\mu(V(w, L, \varepsilon)) & \leq \mu\left(B\left(w, \lambda^{L} \varepsilon\right)\right) \leq \mu\left(B\left(w, \frac{1}{2} Q^{-N-1}\right)\right) \leq \sqrt{2}^{-N+N_{0}+1} . \\
\frac{1}{L} \log \mu(V(w, L, \varepsilon)) & \leq \frac{1}{L}(\log \sqrt{2})\left(-N+N_{0}+1\right) \\
& \leq \frac{\log \lambda}{\log Q} \log \sqrt{2}+\frac{1}{L}(\log \sqrt{2})\left(2+\frac{\log (2 \varepsilon)}{\log Q}+N_{0}+1\right) .
\end{aligned}
$$

By Brin-Katok Theorem [7] and the choice of $w$ in (26), we have that

$$
h_{\mu}(T)=-\lim _{L \rightarrow+\infty} \frac{1}{L} \log \mu(V(w, L, \varepsilon)) \geq \frac{\log \lambda^{-1}}{\log Q} \log \sqrt{2}>0 .
$$

This contradicts the choice of $F$ and $\mu$ in (24). Therefore such non-empty open set $\mathcal{W}$ in (23) does not exist. This implies that the (open) set $\mathcal{O}=\bigcup_{y \in \operatorname{Per}(T)} \mathcal{U}_{y}$ is dense.

Now we prove

3.2. Proposition. For any $\ell \geq 0, \quad t_{\ell+1}^{N}-t_{\ell}^{N} \geq \sqrt{2}^{N-N_{0}-1}$.

Proof: For $N \in \mathbb{N}$, let

$$
\mathbb{A}_{N}:=\left\{(x, y) \in X \times X \mid d(x, y) \leq Q^{-N}\right\} .
$$

From (25) and Statement 3.1, we get 


\subsection{Statement.}

If $N>N_{0}$ and $\left(x_{k}\right)_{k=0}^{p-1}$ is a p-periodic $Q^{-N}$ pseudo-orbit in $\mathcal{O}(q)$ with at most 2 jumps, then there is a $\frac{1}{2} Q^{-N+1}$-return $d\left(x_{i}, x_{j}\right)<\frac{1}{2} Q^{-N+1}$ with $0 \leq i<j<p$. In particular $\left(x_{i}, x_{j}\right) \in \mathbb{A}_{N-1}$

Write $q_{i}:=T^{i}(q)$. From (28), the sequence $\left(q_{k}\right)_{k=t_{\ell}^{N}}^{t_{\ell+1}^{N}-1}$ is a periodic $Q^{-N}$ pseudo-orbit in $\mathcal{O}(q)$ with 1 jump. Therefore there is a $Q^{-N+1}$-return $d\left(q_{i}, q_{j}\right)<\frac{1}{2} Q^{-N+1} \leq Q^{-N+1}$ with $t_{\ell}^{N} \leq i<j<t_{\ell+1}^{N}$. This gives rise to two $Q^{-N+1}$ periodic pseudo-orbits in $\mathcal{O}(q)$ with at most 2 jumps. Namely, $\left(q_{i}, \ldots, q_{j-1}\right)$ and $\left(q_{j}, \ldots, q_{t_{\ell+1}^{N}-1}, q_{t_{\ell}^{N}}, \ldots, q_{i-1}\right)$. Each of them imply a $Q^{-N+2}$ approach... This process will continue as long as $N \geq N_{0}$.
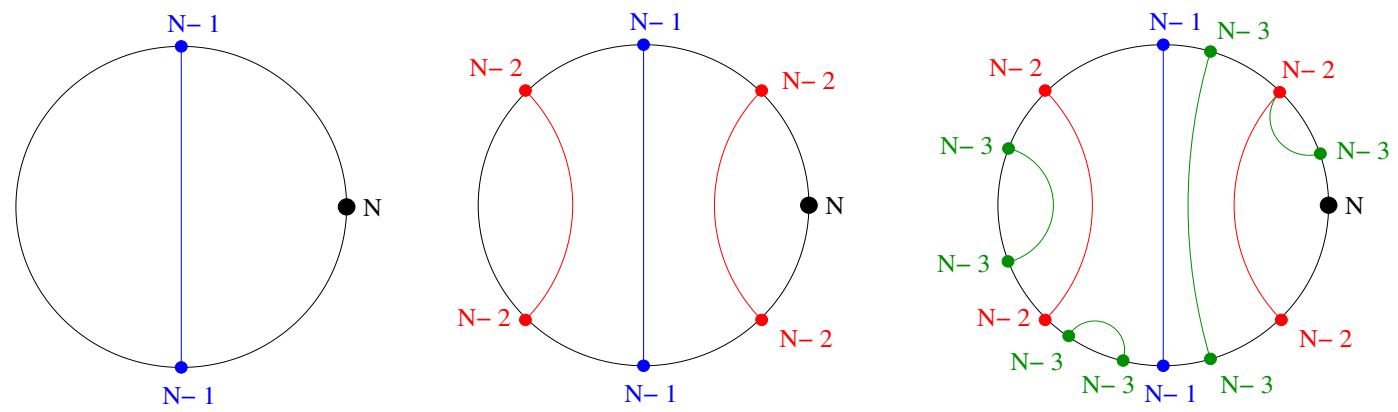

Figure 1 . The disc $\mathbb{D}$, the circle $\mathbb{S}=\partial \mathbb{D}$ and an example of a cascade of returns implied by the inductive process.

It is simpler to show the inductive process in a picture. Draw a circle $\mathbb{S}$ with the elements of the pseudo-orbit $\left(q_{k}\right)_{k=t_{\ell}^{N}}^{t_{\ell+1}^{N}-1}$. Consider a disc $\mathbb{D}$ with boundary $\partial \mathbb{D}=\mathbb{S}$. Inside the disc $\mathbb{D}$, draw a line from $q_{i}$ to $q_{j}$. It may be that $q_{i}=q_{t_{\ell}^{N}}$ but in that case $q_{j} \neq q_{t_{\ell+1}^{N}}$. The line $\ell_{1}=\overline{q_{i} q_{j}}$ separates the disk in two components. Each component is a $Q^{-N+1}$ pseudo-orbit with at most two jumps (one jump of size $\leq Q^{-N+1}$ and possibly another with size $\left.\leq Q^{-N}<Q^{-N+1}\right)$. Thus, each component has at least one $Q^{-N+2}$ return... The interior of the lines in this construction do not intersect.

We will also draw a tree with the returns, in order to see that their number grows exponentially. An example appears in figure 2. The nodes of the tree are the returns implied by Statement 3.3. The height of the node bounds the size of the return. The numbers near a node are the quantity of returns in upper levels of the tree which are adjacent to the return of the node, either at its left or at its right. These numbers are also equal to $-1+$ the quantity of jumps of the two new periodic pseudo-orbits determined by the node. 


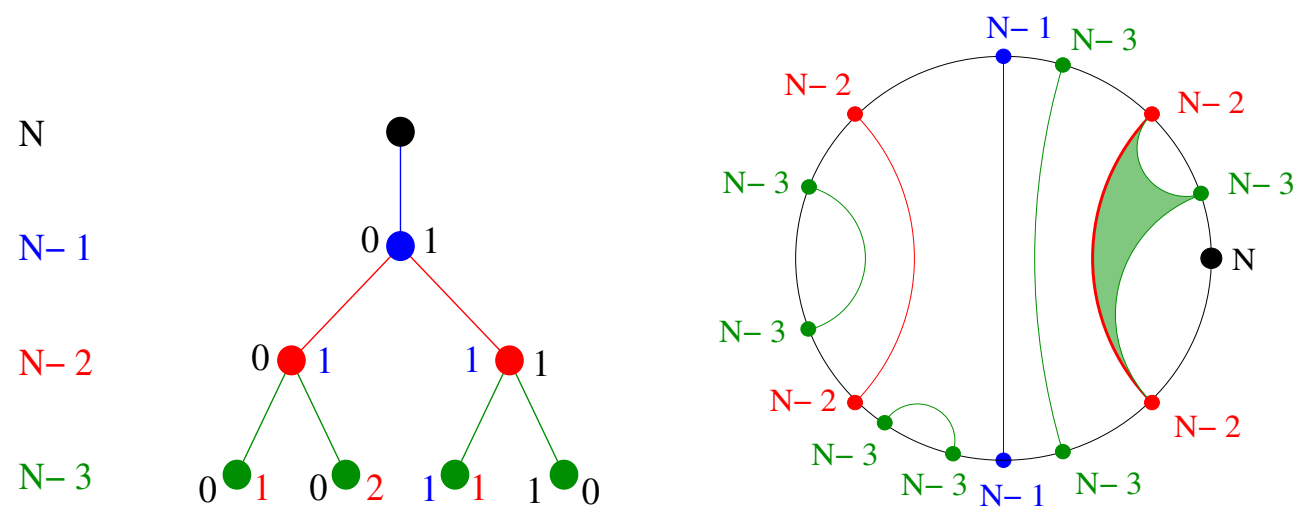

FiguRE 2. An example of a distribution of returns implied by Statement 3.3 and the tree representing it. The shadow is explained in $§ 3.2 .2$ and in Figure 4.

We show how the tree is constructed in the example of figure 2. We begin with a return in $\mathbb{A}_{N}$. This gives a periodic $Q^{-N}$ pseudo-orbit with no other jump. It implies the existence of a return in $\mathbb{A}_{N-1}$. In the tree we draw a vertical line from level $N$ to level $N-1$. At this stage, the line in the circle corresponding to the $\mathbb{A}_{N-1}$ return divides the disk in two components. One side has 1 return in $\mathbb{A}_{N}$ that appears in a previous level in the tree and the other side has 0 returns appearing above in the tree. We write the numbers 0 and 1 at the sides of the node of the tree corresponding to the $\mathbb{A}_{N-1}$ return. The $\mathbb{A}_{N-1}$ return divides the circle in two components. The component at the left is a periodic $Q^{-N+1}$ pseudo-orbit with only one $Q^{-N+1}$ jump, corresponding to the number 0 in the tree. The component at the right is a $Q^{-N+1}$ pseudo-orbit with a $Q^{-N-1}$ jump and also a $Q^{-N}$ jump, and corresponds to the number 1 in the tree in the node at level $N-1$.

Statement 3.3 implies the existence of other returns in $\mathbb{A}_{N-2}$ for both pseudo-orbits. In the right hand side of figure 2 we draw the case in which the pseudo-orbit segment between the $\mathbb{A}_{N-2}$ return contains a $Q^{-N}$ jump. Cutting the $Q^{-N+1}$ pseudo-orbit of the right hand side of the circle at the $\mathbb{A}_{N-2}$ return we obtain two $Q^{-N+2}$ periodic pseudo-orbits. The one at the right has a $Q^{-N}$ jump which appears previously in the tree and the one at the left has a $Q^{-N+1}$ jump appearing previously in the tree. We write the numbers 1 and 1 in the corresponding node of the tree.

We will provide the tree with black nodes $\bullet$ and white (or empty) nodes $\otimes$. The nodes in the tree are associated to the approaches implied by the process. A black node means that at least one of the points in the approach is a point in the pseudo-orbit $\left(q_{t_{\ell}^{N}}, \ldots, q_{t_{\ell+1}^{N}-1}\right)$ 

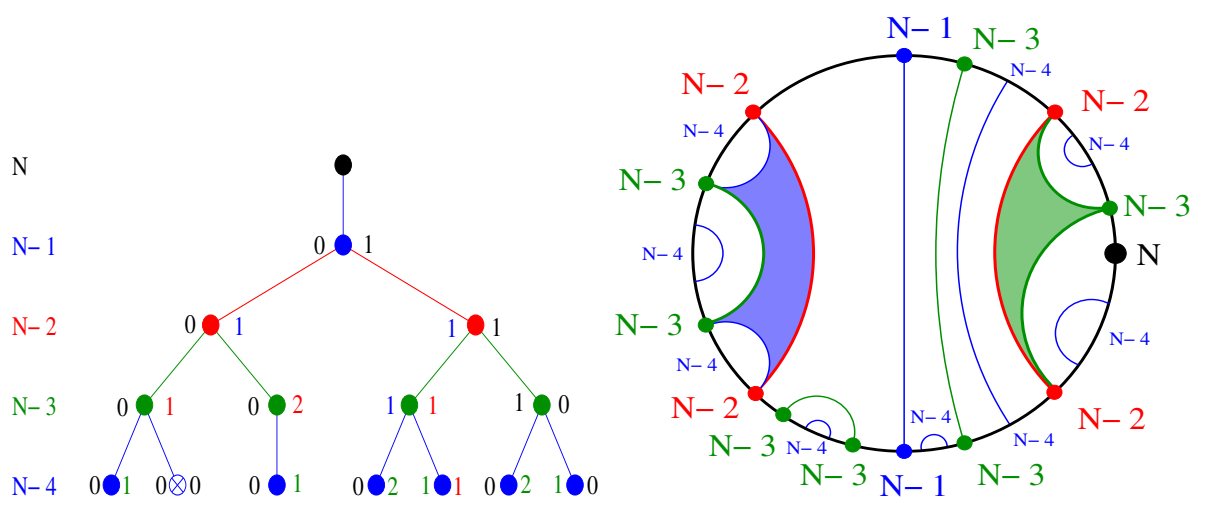

FiguRE 3. This is a possible next step from the example in Figure 2. At level $N-3$ we had a node $0 \bullet 2$ which only issues one branch with label 0 . At level $N-4$ the node $0 \otimes 0$ corresponds to the shadowed region on the left of the disc. This node comes from a branch with label 1, i.e. a periodic specification with 2 jumps. In this case the implied return in $\mathbb{A}_{N-4}$ has both of its points at the jumps of the specification. We put a white (or empty) node $\otimes$ in the tree, signifying that this node (approach) does not count as a new point in the tree, i.e. as another point in the pseudo-orbit $\left(q_{t_{\ell}^{N}}, \ldots, q_{t_{\ell+1}^{N}-1}\right)$ which was not accounted for earlier. We show that in this case both jumps of the specification give two approaches which issue two periodic specifications with only one jump. We write the labels 0 in the node $0 \otimes 0$ meaning that both implied specifications have only one jump. The node $0 \otimes 0$ will issue two branches (with label 0 ). We shadow the cuadrilateral region at the left to be not considered later. After drawing the shadow there remain two white regions in the disc which give two $Q^{-N+4}$ periodic specifications with only one jump that will restore the duplication process.

which did not appear in the previous approaches. So that we have

$$
t_{\ell+1}^{N}-t_{\ell}^{N} \geq \#\{\text { black nodes }\}
$$

The branches of the tree correspond to the new periodic pseudo-orbits implied by the approach at the node which issues the branches. The numbers at the node are associated to the branches issued by the node. The number 2 has no issued branch.

The tree usually duplicates its nodes but we have to be careful of two situations. The first is when an approach implies a periodic pseudo-orbit with more than 2 jumps, i.e. a number 2 (or more) in the tree. For simplicity we have chosen to limit our accounting to at most 2 jumps. In this case Statement 3.3 does not imply the existence of a new approach and we stop the process. In the tree this means that there is no new branch corresponding to a number 2 . We will see that this only happens when the parent node has label $0 \bullet 2$ and that the 0 side does issue a new branch which restarts the duplication process.

The other situation is when at least one point of a new approach is exactly at one of the jumps of the mother pseudo-orbit, see figures 4 and 5 . We will see that in these cases the 

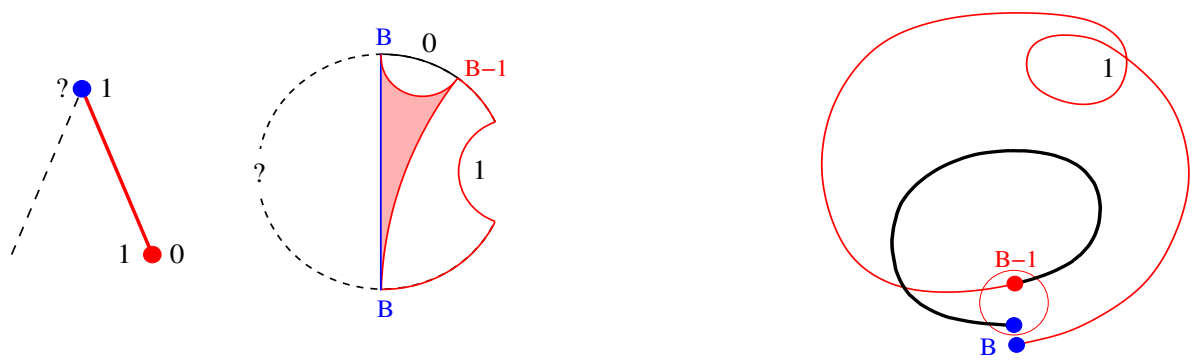

FiguRE 4. If one of the points in the approach implied by Statement 3.3 is one of the jumps of the mother periodic pseudo-orbit we observe that it divides the mother pseudo-orbit in two child pseudo-orbits. We draw lines connecting the ends of these pseudo-orbits and shadow the internal part of the disk $\mathbb{D}$ which does not contain an interval in the circle $\mathbb{S}$.

approach implies two new periodic specifications, and hence two new branches issued from the node corresponding to the approach, which will continue the duplication process. In the case when both points in the approach are at the jumps of previous periodic pseudoorbit, as in Figure 5, both points may have already been accounted for previously in the tree. In this case we put a white (or empty) node $\otimes$ in the tree.
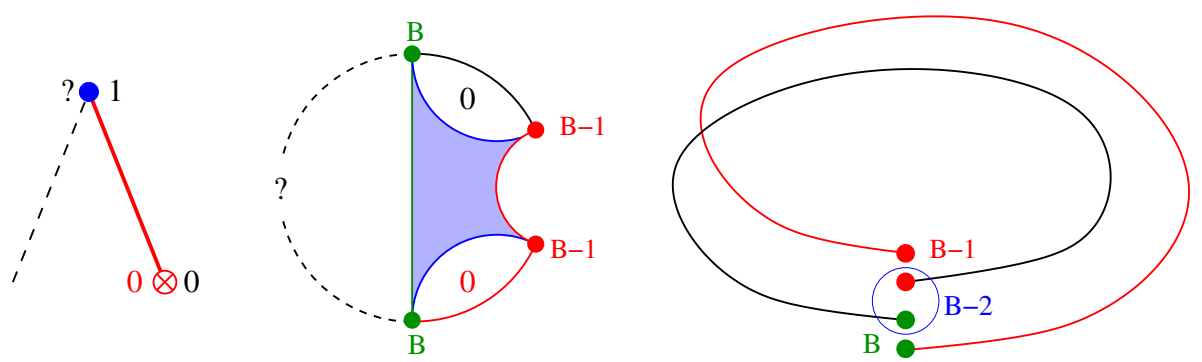

FIGURE 5. If both points of the approach implied by Statement 3.3 are exactly the jumps of the mother periodic pseudo-orbit we observe that it divides the mother pseudo-orbit in two child pseudo-orbits with only one jump. The approach does not necessarily imply a new point in $\left(q_{t_{\ell}^{N}}, \ldots, q_{t_{\ell+1}^{N}}\right)$ which was not accounted for previously in the tree. Therefore we write a white (or empty) node $\otimes$ in the tree. We draw lines connecting the ends of these pseudo-orbits and shadow the internal part of the disk $\mathbb{D}$ which does not contain an interval in the circle $\mathbb{S}$.

We now study the building blocks of the tree. The case of a periodic $Q^{-B}$ pseudo-orbit with only 1 jump is represented in Figure 6, and the case with 2 jumps is in Figure 7.

\subsection{Childs of a periodic pseudo-orbit with 1 jump.}

3.1.1. Case $0 \bullet$ 0. When one point of the approach is at the jump of the mother pseudoorbit. Denote the periodic $Q^{-B}$ pseudo-orbit with 1 jump by $\left(q_{a}, \ldots, q_{b-1}\right), q_{b}=q_{a}$. In 

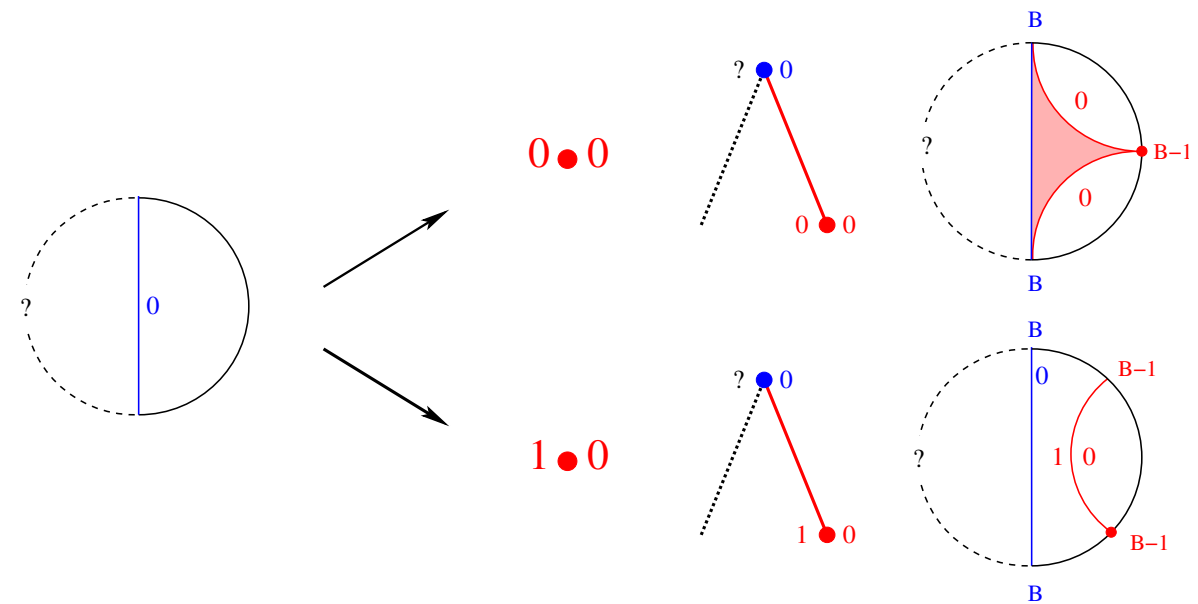

Figure 6. Possible nodes ending a branch with a label 0, i.e. child specifications of a periodic 1-specification with only one jump.

this case the $\frac{1}{2} Q^{-B+1}$ approach is $\left(q_{i}, q_{j}\right)$ with $q_{i}=q_{a}$. Observe that

$$
\begin{aligned}
d\left(T\left(q_{j-1}\right), q_{i}\right) & =d\left(q_{j}, q_{i}\right)<\frac{1}{2} Q^{-B+1} . \\
d\left(T\left(q_{b-1}\right), q_{j}\right) & \leq d\left(T\left(q_{b-1}\right), q_{a}\right)+d\left(q_{a}, q_{j}\right) \\
& =d\left(T\left(q_{b-1}\right), q_{a}\right)+d\left(q_{i}, q_{j}\right) \leq Q^{-B}+\frac{1}{2} Q^{-B+1}<Q^{-B+1} .
\end{aligned}
$$

From (31) we have that $\left(q_{i}, \ldots, q_{j-1}\right)$ is a $Q^{-B+1}$ pseudo-orbit with only 1 jump and from (32) we have that $\left(q_{j}, \ldots, q_{b-1}\right)$ is another $Q^{-B+1}$ pseudo-orbit with only 1 jump. In the disk $\mathbb{D}$ we draw the lines $\overline{q_{a} q_{j}}$ and $\overline{q_{j} q_{b}}$, and also shadow the triangular region limited by the lines $\overline{q_{a} q_{b}}, \overline{q_{a} q_{j}}$ and $\overline{q_{j} q_{b}}$. This shadowed region is treated as a line with a right and left side. The choice of right and left sides may be ambiguous and is left to the reader's will. The two regions left in white in the disk $\mathbb{D}$ correspond to the periodic $Q^{-B+1}$ specifications with only one jump mentioned above. In the tree we label the node with the symbol $0 \bullet 0$. The node is black $\bullet$ because the point $q_{j}$ in the approach $\left(q_{a}, q_{j}\right)$ did not appear before as a node in the tree. This node will have two branches corresponding to the numbers 0 and 0 .

3.1.2. Case $1 \bullet 0$. When both points of the approach are not at the jump of the pseudoorbit. Denote the periodic $Q^{-B}$ pseudo-orbit by $\left(q_{a}, \ldots, q_{b-1}\right), q_{b}=q_{a}$. In this case the $\frac{1}{2} Q^{-B+1}$ approach is $\left(q_{i}, q_{j}\right)$ with $a<i<j<b$; it implies two daughter periodic $Q^{-B+1}$ pseudo-orbits: $\left(q_{a}, \ldots, q_{i-1}, q_{j}, \ldots, q_{b-1}\right)$ with 2 jumps and $\left(q_{i}, \ldots, q_{j-1}\right)$ with only 1 jump. In the tree we label the node as $1 \bullet 0$. The numbers 1 and 0 correspond to the new implied $Q^{-B+1}$ pseudo-orbits with 2 and 1 jumps respectively. The node issues two branches 
corresponding to the numbers 1 and 0 . The node is black $\bullet$ because the approach $\left(q_{i}, q_{j}\right)$ has one of its points (in fact both points) which did not appear before in the nodes of the tree.

$0 \otimes 0$
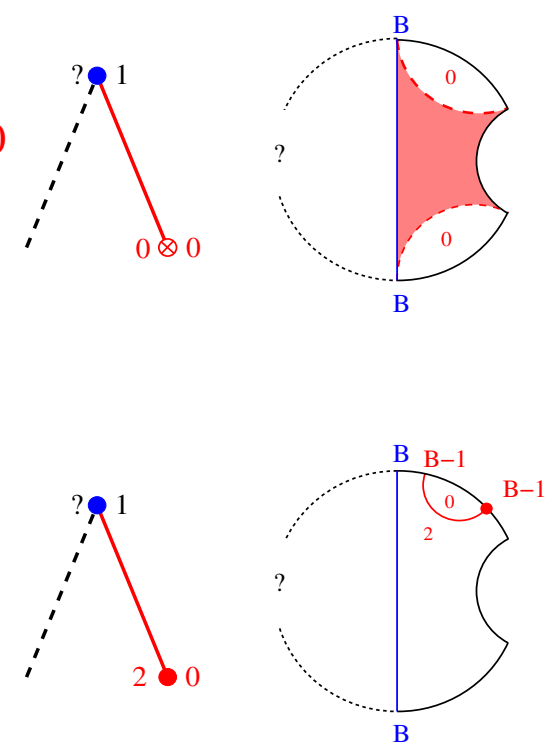
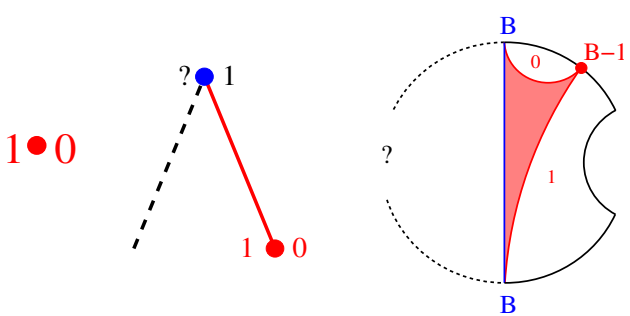

$1 \bullet 1$
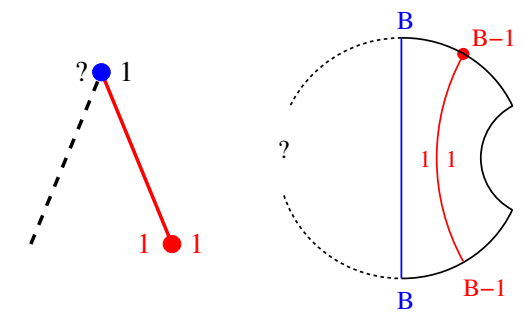

FigURE 7. Possible nodes ending a branch with a label 1, i.e. child specifications of a periodic 1-specification with two jumps.

\subsection{Childs of a periodic pseudo-orbit with 2 jumps.}

3.2.1. Case $0 \otimes 0$. When both points in the approach are the jumps of the pseudo-orbit. Denote the mother $Q^{-B}$ pseudo-orbit by $\left(q_{a}, \ldots, q_{b-1}, q_{c}, \ldots, q_{d-1}\right)$ with 2 jumps at $q_{b}=q_{c}$ and $q_{d}=q_{a}$. In this case the $\frac{1}{2} Q^{-B+1}$ approach is $\left(q_{i}, q_{j}\right)=\left(q_{a}, q_{c}\right)$. Observe that

$$
\begin{aligned}
& d\left(T\left(q_{b-1}\right), q_{a}\right) \leq d\left(T\left(q_{b-1}\right), q_{c}\right)+d\left(q_{c}, q_{a}\right) \leq Q^{-B}+\frac{1}{2} Q^{-B+1}<Q^{-B+1} . \\
& d\left(T\left(q_{d-1}\right), q_{c}\right) \leq d\left(T\left(q_{d-1}\right), q_{a}\right)+d\left(q_{a}, q_{c}\right) \leq Q^{-B}+\frac{1}{2} Q^{-B+1}<Q^{-B+1} .
\end{aligned}
$$

By (33) we have that $\left(q_{a}, \ldots, q_{b-1}\right)$ is a periodic $Q^{-B+1}$ pseudo-orbit with only one jump. By $(34),\left(q_{c}, \ldots, q_{d-1}\right)$ is another periodic $Q^{-B+1}$ pseudo-orbit with only one jump. The points in the approach $\left(x_{a}, x_{c}\right)$ may have both appeared before as nodes (i.e. approaches) in the tree, so we write a white (or empty) node $\otimes$. The label of the node is $0 \otimes 0$ because both child pseudo-orbits have only one jump. The node issues two branches corresponding to the numbers 0 and 0 . In the disc we draw the lines $\overline{q_{a} q_{b}}$ and $\overline{q_{c} q_{d}}$ corresponding to the new approaches and shadow the quadrilateral region limited by these lines and the previously drawn lines $\overline{q_{b} q_{c}}$ and $\overline{q_{a} q_{d}}$. 
3.2.2. Case $0 \bullet 1$. When one point of the approach is one of the jumps of the pseudoorbit. Denote the $Q^{-B}$ pseudo-orbit by $\left(q_{a}, \ldots, q_{b-1}, q_{c}, \ldots, q_{d-1}\right)$ with jumps at $q_{b}$ and $q_{d}$. We can assume that in this case $q_{a}$ is one of the points in the $\frac{1}{2} Q^{-B+1}$ approach $\left(q_{i}, q_{j}\right)=\left(q_{a}, q_{j}\right)$. We will further assume that $a<j<b$ as in Figure 7 , the other case $c<j<d$ is similar. The point $q_{j}$ has not appeared before in the tree, so we put a black node $\bullet$. We have that $\left(q_{a}, \ldots, q_{j-1}\right)$ is a $Q^{-B+1}$ pseudo-orbit with only 1 jump, which gives a number $0 \bullet$ in the tree. Observe that

$$
\begin{aligned}
d\left(T\left(q_{d-1}\right), q_{j}\right) & \leq d\left(T\left(q_{d-1}\right), q_{a}\right)+d\left(q_{a}, q_{j}\right) \\
& \leq d\left(T\left(q_{d-1}\right), q_{a}\right)+d\left(q_{i}, q_{j}\right) \leq Q^{-B}+\frac{1}{2} Q^{-B+1}<Q^{-B+1} .
\end{aligned}
$$

Therefore $\left(q_{j}, \ldots, q_{b-1}, q_{c}, \ldots, q_{d-1}\right)$ is a periodic $Q^{-B+1}$ pseudo-orbit with two jumps. We write the label 1 in the node $0 \bullet 1$. The node issues two branches corresponding to the numbers 0 and 1 . In the disc we draw the lines $\overline{q_{a} q_{j}}$ and $\overline{q_{j} q_{d}}$. We shadow the triangular region bounded by the lines $\overline{q_{d} q_{a}}, \overline{q_{a} q_{j}}$ and $\overline{q_{j} q_{d}}$. We treat the shadowed region as a line with right and left sides, at the choice of the reader. The white regions left by the shadow are the two specifications with 1 and 2 jumps described above.

3.2.3. Case $0 \bullet 2$. When both of the points of the approach are in the interior of one segment of the pseudo-orbit. Let $\left(q_{a}, \ldots, q_{b-1}, q_{c}, \ldots, q_{d-1}\right)$ be the $Q^{-B}$ pseudo-orbit with jumps at $q_{b}$ and $q_{d}$. We can assume that the approach $\left(q_{i}, q_{j}\right)$ is in interior of the first segment $\left(q_{a}, \ldots, q_{b-1}\right)$ of the pseudo-orbit, i.e. $a<i<j<b$. Both points of the approach did not appear before in the tree so this is a black node •. The segment $\left(q_{i}, \ldots, q_{j-1}\right)$ is a periodic $Q^{-B+1}$ pseudo-orbit with only one jump, which gives a number 0 in the node $0 \bullet$. The rest of the pseudo-orbit is a periodic pseudo-orbit with 3 jumps: $\left(q_{a}, \ldots, a_{i-1}, q_{j}, \ldots, q_{b-1}, q_{c}, \ldots, q_{d-1}\right)$. We write a number 2 in the node $0 \bullet 2$. We stop the process at the pseudo-orbit with 3 jumps. The node will issue only one branch corresponding to the number 0 .

3.2.4. Case 1•1. When the points in the approach are in the interior of both segments of the pseudo-orbit. Let $\left(q_{a}, \ldots, q_{b-1}, q_{c}, \ldots, q_{d-1}\right)$ be the periodic $Q^{-B}$ pseudo-orbit. The indices of the approach $\left(x_{i}, x_{j}\right)$ satisfy $a<i<b<c<j<d$. Both points of the approach did not appear before in the tree, so the node is black •. Both $\left(q_{a}, \ldots, q_{i-1}, q_{j}, \ldots, q_{d-1}\right)$ and $\left(q_{i}, \ldots, q_{b-1}, q_{c}, \ldots, q_{j-1}\right)$ are periodic $Q^{-B+1}$ pseudo-orbits with 2 jumps, thus the label of the node is $1 \bullet 1$. This node $1 \bullet 1$ issues two branches, each one with the number 1. 

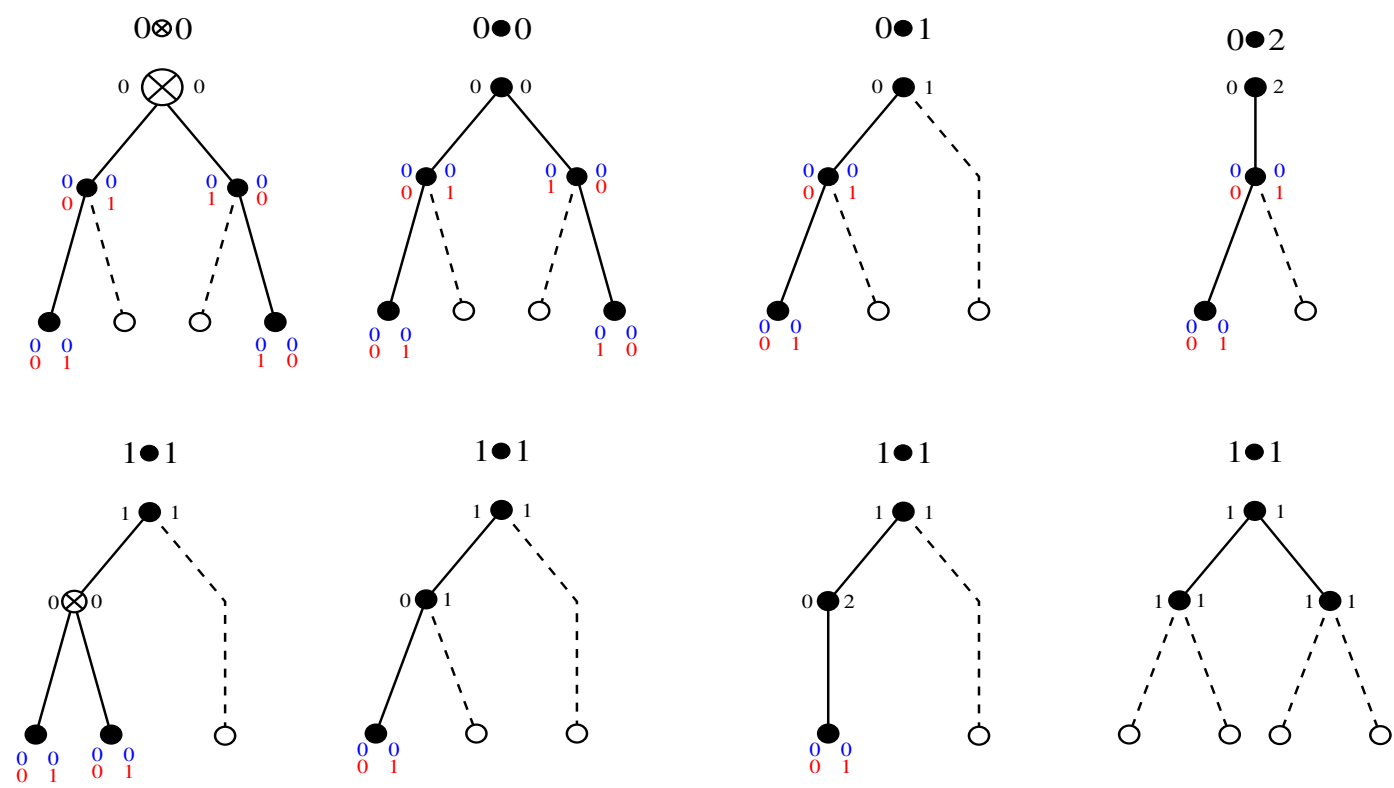

FigurE 8. This figure shows that the tree satisfies Claim 3.4. The dots o mean that we don't know if it is a white or black node. The dotted lines mean that we know that there is at least one branch, but we know neither the label of the branch nor the label and color of the ending node. The long dashed lines mean that the corresponding subtree has at least one ending node. The pictures use the fact from $\S 3.1$ and Figure 6 that a branch with label 0 can only end in nodes with labels $0 \bullet 0$ or $0 \bullet 1$. And in both of these cases the node has again at least one new branch with label 0 . All of the pictures satisfy Claim 3.4: i.e. at least two black dots in levels $N-1, N-2$ and at least two nodes, black or white, at the ending level $N-2$.

The tree is built from the nodes described in $\S 3.1$ and $\S 3.2$ which also appear in Figure 6 and Figure 7 respectively. In order to obtain the estimate in Proposition 3.2 it is enough to show that at any consecutive pair of levels, the tree duplicates its number of black nodes, because in that case we have

$$
t_{\ell+1}^{N}-t_{\ell}^{N} \geq \#\{\text { black nodes }\} \geq 2^{\frac{N-N_{0}-1}{2}} .
$$

To obtain the duplication it is enough to show the following

3.4. Claim: At each node, black or white, in level $N>N_{0}+1$ the sub-tree below the node has at least two black nodes at levels $N-1$ and $N-2$ (added together) and also at level $N-2$ the subtree of the node has at least two nodes, black or white.

Because then at any two consecutive levels $N-1, N-2$ the number of black nodes duplicates the number of nodes at level $N$ and also the total number of nodes at level $N-2$ duplicates the number of nodes at level $N$. 
In Figure 8 we check that Claim 3.4 is true. The figures take advantage (from $\S 3.1$ and Figure 6) that a branch with label 0 ends in a black node with label $0 \bullet 0$ or $0 \bullet 1$. In both cases the node has at least one branch with label 0 again. The dots $\circ$ mean that we don't know if the node is black • or white $\otimes$. In $\S 3.1$ and $\S 3.2$ (or Fig. 6 and Fig. 7) we see that all the labels for the nodes have at least one number smaller than 2 . This implies that every node issues at least one branch. In Figure 8 there are some long dashed lines which mean that we know that there is at least one branch and at least one ending node, but we don't pay attention to more details.

The subtrees from a node $1 \bullet 1$ are drawn in the lower line in Figure 8 . They are ordered by the first child node at the left hand side. The first three cases in the second row show that a subtree from a node $1 \bullet 1$ which has a child with label either $0 \otimes 0,0 \bullet 1$ or $0 \bullet 2$ satisfies the Claim 3.4. The last case is a node $1 \bullet 1$ with one left child node $1 \bullet 1$. For the right branch, we have already seen that if the right node is $0 \otimes 0,0 \bullet 1$ or $0 \bullet 2$ then the subtree satisfies Claim 3.4. It only remains the case in which the right node is also $1 \bullet 1$. Figure 8 shows that this last case also satisfies Claim 3.4.

This completes the proof of Proposition 3.2.

\section{Appendix A. Zero Entropy.}

In this appendix we prove Ian Morris Theorem 1.2. The published version was written for symbolic dynamics. We need two lemmas.

A.1. Lemma. Let $a_{1}, \ldots, a_{n}$ be non-negative real numbers, and let $A=\sum_{i=1}^{n} a_{i} \geq 0$. Then

$$
\sum_{i=1}^{n}-a_{i} \log a_{i} \leq 1+A \log n
$$

where we use the convention $0 \log 0=0$.

Proof: Applying Jensen's inequality to the concave function $x \mapsto-x \log x$ yields

$$
\frac{1}{n} \sum_{i=1}^{n}-a_{i} \log a_{i} \leq-\left(\frac{1}{n} \sum_{i=1}^{n} a_{i}\right) \log \left(\frac{1}{n} \sum_{i=1}^{n} a_{i}\right)=-\frac{A}{n} \log A+\frac{A}{n} \log n
$$

from which the result follows.

A.2. Lemma. Let $f \in \operatorname{Lip}(X, \mathbb{R})$ and suppose that $\mathcal{M}_{\max }(f)=\{\mu\}$ for some $\mu \in \mathcal{M}(T)$. Then there is $C>0$ such that for every $\nu \in \mathcal{M}(T)$,

$$
-\alpha(f)-C \int d(x, K) d \nu \leq \int f d \nu
$$


where $K=\operatorname{supp} \mu$.

Proof: By Proposition 2.2 and Lemma 2.1.3.ii there exists $g \in \operatorname{Lip}(X, \mathbb{R})$ such that $f+g-g \circ T \leq-\alpha(f)$. Define $\tilde{f}=f+g-g \circ T$. Since $\mu \in \mathcal{M}_{\max }(f)$,

$$
\int \tilde{f} d \mu=\int f d \mu=-\alpha(f) \quad \text { and } \quad \tilde{f} \leq-\alpha(f) .
$$

Since $\tilde{f}$ is continuous, it follows that $\tilde{f}(z)=-\alpha(f)$ for every $z \in K=\operatorname{supp} \mu$. Let $C=\operatorname{Lip}(\tilde{f})$. Given $x \in X$, let $z \in K$ be such that $d(x, z)=d(x, K)$. We have that

$$
\tilde{f}(x) \geq \tilde{f}(z)-C d(x, z)=-\alpha(f)-C d(x, K)
$$

from which the result follows.

\subsection{Theorem (Morris [18]).}

Let $X$ be a compact metric space and $T: X \hookleftarrow$ an expanding map. There is a residual set $\mathcal{G} \subset \operatorname{Lip}(X, \mathbb{R})$ such that if $F \in \mathcal{G}$ then there is a unique $F$-maximizing measure and it has zero metric entropy.

A.3. Remark. By the linearity of the integral, or by the characterization of maximizing measures in Lemma 2.1-2.(iii), the ergodic components of a maximizing measure are also maximizing. Therefore the unique maximizing measure in Theorem 1.2 is ergodic. In fact the map $\left.T\right|_{\operatorname{supp}(\mu)}$ is uniquely ergodic.

\section{Proof of Theorem 1.2:}

For $p \geq 1$ let $\mathcal{M}^{p}(T)$ be the set of invariant probabilities supported on a periodic orbit of period smaller or equal to $p$. In this appendix we will identify a periodic orbit $\left\{z, T z, \ldots, T^{p-1} z\right\}$ with the corresponding invariant measure $\mu=\frac{1}{p} \sum_{i=0}^{p-1} \delta_{T^{i} z}$.

Let

$$
e_{0}>0, \quad 0<\lambda<1
$$

be such that for every $x \in X$ the branches of the inverses of $T$ at $x$ are well defined, injective, and are $\lambda$-contractions on the ball $B\left(x, \varepsilon_{0}\right)$ of radius $e_{0}$ centered at $x$.

Let

$$
\mathcal{E}_{\gamma}:=\left\{f \in \operatorname{Lip}(X, \mathbb{R}) \mid h(\mu)<2 \gamma h_{\mathrm{top}}(T) \quad \forall \mu \in \mathcal{M}_{\max }(f)\right\} .
$$

By Theorem 1.1 the set

$$
\mathcal{O}=\left\{f \in \operatorname{Lip}(X, \mathbb{R}) \mid \# \mathcal{M}_{\max }(f)=1\right\}
$$

is residual. 
It is enough to prove that $\mathcal{E}_{\gamma}$ is open and dense for every $\gamma>0$, for then the set

$$
\mathcal{G}=\mathcal{O} \cap \bigcap_{n \in \mathbb{N}} \mathcal{E}_{\frac{1}{n}}
$$

satisfies the requirements of the Theorem.

Step 1. $\mathcal{E}_{\gamma}$ is open.

Suppose that $f \in \operatorname{Lip}(X, \mathbb{R}), f_{n} \in \operatorname{Lip}(X, \mathbb{R}) \backslash \mathcal{E}_{\gamma}$ and $\lim _{n} f_{n}=f$ in $\operatorname{Lip}(X, \mathbb{R})$. Then there are $\nu_{n} \in \mathcal{M}_{\max }\left(f_{n}\right)$ with $h\left(\nu_{n}\right) \geq 2 \gamma h_{\text {top }}(T)$. Taking a subsequence if necessary, we may assume that $\nu_{n} \rightarrow \nu \in \mathcal{M}(T)$. For any $\mu \in \mathcal{M}(T)$ we have that

$$
\int f d \mu-\left\|f-f_{n}\right\|_{\infty} \leq \int f_{n} d \mu \leq \int f_{n} d \nu_{n} \leq \int f d \nu_{n}+\left\|f-f_{n}\right\|_{\infty} .
$$

Taking $\lim _{n}$ we get that $\int f d \mu \leq \int f d \nu$ for any $\mu \in \mathcal{M}(T)$ and hence $\nu \in \mathcal{M}_{\text {max }}(T)$. Since the map $m \mapsto h(m)$ is upper semicontinuous (see e.g. Walters [22, Theorem 8.2]) we have that $h(\nu) \geq 2 \gamma h_{\text {top }}(T)$. Therefore $f \in \operatorname{Lip}(X, \mathbb{R}) \backslash \mathcal{E}_{\gamma}$. We conclude that $\operatorname{Lip}(X, \mathbb{R}) \backslash \mathcal{E}_{\gamma}$ is closed and then $\mathcal{E}_{\gamma}$ is open.

Step 2. We have to prove that $\mathcal{E}_{\gamma}$ intersects every non-empty open set. Let $\mathcal{U} \subset$ $\operatorname{Lip}(X, \mathbb{R})$ be open and non-empty. By Theorem 1.1 there is $f \in \mathcal{U}$ such that $\mathcal{M}_{\max }(f)$ has only one element $\mu$. If $\mu$ is a periodic orbit then $f \in \mathcal{E}_{\gamma} \cap \mathcal{U}$ and we are done. Otherwise, since by Lemma 2.1-2.(iii) any measure in $\operatorname{supp}(\mu)$ would also be maximizing, we have that $K:=\operatorname{supp}(\mu)$ does not contain a periodic orbit. By Lemma A.2 there is a real number $C>0$ and a compact invariant set $K$ such that for every $\nu \in \mathcal{M}(T)$

$$
-\alpha(f)-C \int d(x, K) d \nu \leq \int f d \nu
$$

and such that $K$ does not contain a periodic orbit.

Let $\beta>0$ be small enough that $f+g \in \mathcal{U}$ whenever

$$
\|g\|_{0}+\operatorname{Lip}(g) \leq(\operatorname{diam} X+1) \beta .
$$

We will construct a sequence of approximating functions such that $f_{n} \in \mathcal{U} \cap \mathcal{E}_{\gamma}$ for $n$ large enough. In the next two steps we choose a sequence of periodic orbits which will be used in the construction.

Step 3.

A.4. Claim: Given any $0<\theta<1$, there is a sequence of integers $\left(m_{n}\right)_{n}$ and a sequence of periodic orbits $\mu_{n} \in \mathcal{M}^{n}(T)$ such that

$$
\int d(x, K) d \mu_{n}=o\left(\theta^{m_{n}}\right) \quad \text { and } \quad \lim _{n \rightarrow \infty} \frac{\log n}{m_{n}}=0 .
$$


Proof of the Claim. By a theorem of Bressaud and Quas [6, Corollary 3 and Theorem 4] for every $k>0$

$$
\lim _{n \rightarrow+\infty} n^{k}\left(\inf _{\mu \in \mathcal{M}^{n}(T)} \int d(x, K) d \mu\right)=0
$$

Indeed recall that using a Markov partition (cf. Ruelle [20, $\$ 7.29]$ ) the map $T$ is Hölder continuously semi-conjugate to a subshift of finite type. This is enough to obtain estimate (38) (see the proof of Corollary 3 in Bressaud and Quas [6]).

From (38) there exists a sequence of periodic orbits $\mu_{n} \in \mathcal{M}^{n}(T)$ such that

$$
\lim _{n \rightarrow+\infty} n^{k} \int d(x, K) d \mu_{n}=0 .
$$

Define

$$
r_{n}:=\log _{\theta}\left(\int d(x, K) d \mu_{n}\right)
$$

Since

$$
\theta^{r_{n}} \leq n^{k} \theta^{r_{n}} \leq 1 \quad \Longleftrightarrow \quad 0 \geq \frac{\log _{\theta} n}{r_{n}} \geq-\frac{1}{k},
$$

we have that $r_{n}^{-1} \log _{\theta} n \rightarrow 0$. Define $m_{n}:=\left\lfloor\frac{1}{2} r_{n}\right\rfloor$, then $m_{n}^{-1} \log _{\theta} n \rightarrow 0$ and

$$
\int d(x, K) d \mu_{n}=\theta^{r_{n}} \leq \theta^{m_{n}+\frac{1}{2} r_{n}}=o\left(\theta^{m_{n}}\right)
$$

as required.

Step 4. Using (35) fix

$$
0<\theta<\min \left\{e_{0}, \lambda, e_{0} \operatorname{Lip}(T)^{-1}\right\}
$$

Choose $m_{n}$ and $\mu_{n}$ as in Claim A.4. Define $L_{n}:=\operatorname{supp} \mu_{n}$.

A.5. Claim: There is $N_{\gamma}>0$ such that when $n \geq N_{\gamma}$

$$
\nu\left(\left\{x \in X \mid d\left(x, L_{n}\right) \geq \theta^{m_{n}}\right\}\right)>\gamma
$$

for every invariant measure $\nu \in \mathcal{M}(T)$ such that $h(\nu) \geq 2 \gamma h_{\mathrm{top}}(T)$.

\section{Proof of the Claim.}

Recall that a Markov partition for $T$ is a finite collection of sets $S_{i}$ which cover $X$ such that

(a) $S_{i}=\overline{\operatorname{int} S_{i}}$.

(b) If $i \neq j$ then int $S_{i} \cap \operatorname{int} S_{j}=\emptyset$.

(c) $f\left(S_{i}\right)$ is a union of sets $S_{j}$. 
Ruelle [20, §7.29] proves that for expanding maps there are Markov partitions of arbitrarily small diameter. Let $\mathbb{P}$ be a Markov partition with $\operatorname{diam} \mathbb{P}<e_{0}$. The elements of the partition

$$
\mathbb{P}^{(n)}:=\bigvee_{i=0}^{n-1} T^{-i} \mathbb{P}=\left\{\bigcap_{i=0}^{n-1} A_{i} \mid A_{i} \in T^{-i} \mathbb{P}\right\}
$$

have diameter smaller than $\lambda^{n-1} e_{0}$ and contain an open set. Then the partition $\mathbb{P}$ is generating because the $\sigma$-algebra

$$
\mathbb{P}^{\infty}=\sigma\left(\cup_{n} \mathbb{P}^{(n)}\right)=\operatorname{Borel}(X) .
$$

contains all the open sets. ${ }^{2}$ Therefore (cf. Walters [22, Thm. 4.18]) for every invariant measure $\nu \in \mathcal{M}(T)$,

$$
h(\nu)=\inf _{k} \frac{1}{k} \sum_{A \in \mathbb{P}^{(k)}}-\nu(A) \log \nu(A) .
$$

From the definition of topological entropy using covers (cf. Walters [22, §7.1]) we have that

$$
\lim _{k \geq 1} \frac{1}{k} \log \# \mathbb{P}^{(k)} \leq h_{\mathrm{top}}(T)
$$

Choose $N_{\gamma}$ large enough such that for all $n \geq N_{\gamma}$

$$
\frac{2+\log \# \mathbb{P}}{m_{n}}+\frac{\log n}{m_{n}}+\frac{\gamma}{m_{n}} \log \# \mathbb{P}^{\left(m_{n}\right)}<2 \gamma h_{\mathrm{top}}(T) .
$$

Let $\nu \in \mathcal{M}(T)$ and suppose that

$$
\nu\left(\left\{x \in X \mid d\left(x, L_{n}\right) \geq \theta^{m_{n}}\right\}\right) \leq \gamma
$$

for some $n \geq N_{\gamma}$. We will show that necessarily $h(\nu)<2 \gamma h_{\mathrm{top}}(\nu)$.

Let

$$
W_{n}:=\left\{A \in \mathbb{P}^{\left(m_{n}\right)} \mid d\left(x, L_{n}\right)<\theta^{m_{n}} \text { for some } x \in A\right\} .
$$

From (41),

$$
\tilde{\gamma}_{n}:=\sum_{A \in \mathbb{P}^{\left(m_{n}\right)} \backslash W_{n}} \nu(A) \leq \gamma
$$

\footnotetext{
${ }^{2}$ The star of a point $x$ in $\mathbb{P}^{(n)}, S(x)=\cup\left\{A \in \mathbb{P}^{(n)} \mid x \in A\right\}$, contains at most \#P elements, has diameter $\leq 2 \lambda^{n-1} e_{0}$ and contains a neighborhood of the point $x$. Therefore any open set in $X$ is a union of (countably many) elements of $\cup_{n} \mathbb{P}^{(n)}$.
} 
Using lemma A.1 we have that

$$
\begin{aligned}
h(\nu) & \leq \frac{1}{m_{n}} \sum_{A \in W_{n}}-\nu(A) \log \nu(A)+\frac{1}{m_{n}} \sum_{A \in \mathbb{P}^{\left(m_{n}\right)} \backslash W_{n}}-\nu(A) \log \nu(A) \\
& \leq \frac{1}{m_{n}}\left(1+\left(1-\tilde{\gamma}_{n}\right) \log \# W_{n}\right)+\frac{1}{m_{n}}\left(1+\gamma \log \# \mathbb{P}^{\left(m_{n}\right)}\right) .
\end{aligned}
$$

Let $g$ be a branch of the inverse of $T^{m_{n}}$. If $x, y$ are in the domain of $g$, we have that

$$
d(g(x), g(y)) \geq \operatorname{Lip}(T)^{-m_{n}} d\left(T^{m_{n}}(g(x)), T^{m_{n}}(g(y))\right) \geq \operatorname{Lip}(T)^{-m_{n}} d(x, y) .
$$

Using (39), observe that since $\theta^{m_{n}}<e_{0} \operatorname{Lip}(T)^{-m_{n}}$ for any $y \in L_{n}$ there is a branch $g$ of the inverse of $T^{m_{n}}$ such that the ball

$$
B\left(y, \theta^{m_{n}}\right) \subseteq g\left(B\left(T^{m_{n}} y, e_{0}\right)\right)
$$

Since $\mathbb{P}$ is a Markov partition with $\operatorname{diam} \mathbb{P}<e_{0}$,

$$
\mathbb{P}^{\left(m_{n}\right)}=\left\{g(A) \mid A \in \mathbb{P}, \quad g \text { is branch of } T^{-m_{n}}\right\} .
$$

Therefore the ball $B\left(y, \theta^{m_{n}}\right)$ intersects at most \#P elements of $\mathbb{P}^{\left(m_{n}\right)}$ because by applying $T^{m_{n}}$

$$
\#\left\{B \in \mathbb{P}^{\left(m_{n}\right)} \mid B \cap B\left(y, \theta^{m_{n}}\right) \neq \emptyset\right\} \leq \#\left\{A \in \mathbb{P} \mid A \cap B\left(T^{m_{n}} y, e_{0}\right) \neq \emptyset\right\} \leq \# \mathbb{P} .
$$

Since $L_{n}$ has at most $n$ elements, $\# W_{n} \leq n \# \mathbb{P}$. Thus from (42) and (40) we have that

$$
\begin{aligned}
h(\nu) & \leq \frac{1}{m_{n}}\left(1+\left(1-\tilde{\gamma}_{n}\right) \log n \# \mathbb{P}\right)+\frac{1}{m_{n}}\left(1+\gamma \log \# \mathbb{P}^{\left(m_{n}\right)}\right) . \\
& \leq \frac{2+\log \# \mathbb{P}}{m_{n}}+\frac{\log n}{m_{n}}+\frac{\gamma}{m_{n}} \log \# \mathbb{P}^{\left(m_{n}\right)}<2 \gamma h_{\mathrm{top}}(T) .
\end{aligned}
$$

This proves the claim.

Step 5. We now complete the proof. Define a sequence of functions $f_{n} \in \operatorname{Lip}(X, \mathbb{R})$ by

$$
f_{n}(x)=f(x)-\beta d\left(x, L_{n}\right),
$$

where $L_{n}=\operatorname{supp} \mu_{n}$ as above. From the definition of $\beta$ in (37) we have that $f_{n} \in \mathcal{U}$ for each $n \geq 1$. From Claim A.4 in step 3 we have that

$$
\int d(x, K) d \mu_{n}=o\left(\theta^{m_{n}}\right)
$$

and from Claim A.5 in step 4 it follows that when $n$ is sufficiently large,

$$
\int d\left(x, L_{n}\right) d \nu \geq \theta^{m_{n}} \nu\left(\left\{x \in X \mid d\left(x, L_{n}\right) \geq \theta^{m_{n}}\right\}\right) \geq \gamma \theta^{m_{n}}
$$

for all $\nu \in \mathcal{M}(T)$ such that $h(\nu) \geq 2 \gamma h_{\text {top }}(T)$. 
We may therefore choose $n$ such that $\beta \int d\left(x, L_{n}\right) d \nu>C \int d(x, K) d \mu_{n}$ for every $\nu \in$ $\mathcal{M}(T)$ such that $h(\nu) \geq 2 \gamma h_{\text {top }}(T)$. It follows that for every such measure $\nu$

$$
\begin{aligned}
\int f_{n} d \nu & =\int f d \nu-\beta \int d\left(x, L_{n}\right) d \nu \\
& <-\alpha(f)-C \int d(x, K) d \mu_{n} \\
& \leq \int f d \mu_{n}=\int f_{n} d \mu_{n} \leq-\alpha\left(f_{n}\right),
\end{aligned}
$$

where we have applied (36) and (43). We have shown that if $\nu \in \mathcal{M}(T)$ and $h(\nu) \geq$ $2 \gamma h_{\text {top }}(T)$, then $\nu \notin \mathcal{M}_{\max }\left(f_{n}\right)$, and therefore $f_{n} \in \mathcal{E}_{\gamma} \cap U$. We conclude that $\mathcal{E}_{\gamma}$ is dense in $\operatorname{Lip}(X, \mathbb{R})$ and the theorem is proved.

\section{REFERENCES}

[1] A. T. Baraviera, R Leplaideur, and A. O. Lopes, Ergodic optimization, zero temperature limits and the max-plus algebra, IMPA, Rio de Janeiro, 2013, $29^{0}$ Coloquio Brasileiro de Matematica.

[2] Thierry Bousch, Le poisson n'a pas d'arêtes, Ann. Inst. H. Poincaré Probab. Statist. 36 (2000), no. 4, 489-508.

[3] __ La condition de Walters, Ann. Sci. École Norm. Sup. (4) 34 (2001), no. 2, 287-311.

[4] Rufus Bowen, Equilibrium states and the ergodic theory of Anosov diffeomorphisms, Springer-Verlag, Berlin, 1975, Lecture Notes in Mathematics, Vol. 470.

[5] Julien Brémont, Gibbs measures at temperature zero, Nonlinearity 16 (2003), no. 2, 419-426.

[6] Xavier Bressaud and Anthony Quas, Rate of approximation of minimizing measures, Nonlinearity 20 (2007), no. 4, 845-853.

[7] Michael Brin and Anatole Katok, On local entropy., Geometric dynamics, Proc. int. Symp., Rio de Janeiro/Brasil 1981, Lect. Notes Math. 1007, 1983, pp. 30-38.

[8] J.-R. Chazottes, J.-M. Gambaudo, and E. Ugalde, Zero-temperature limit of one-dimensional Gibbs states via renormalization: the case of locally constant potentials, Ergodic Theory Dynam. Systems 31 (2011), no. 4, 1109-1161.

[9] Jean-René Chazottes and Michael Hochman, On the zero-temperature limit of Gibbs states, Comm. Math. Phys. 297 (2010), no. 1, 265-281.

[10] G. Contreras, A. O. Lopes, and $\mathrm{Ph}$. Thieullen, Lyapunov minimizing measures for expanding maps of the circle, Ergodic Theory Dynam. Systems 21 (2001), no. 5, 1379-1409.

[11] Albert Fathi, Théorème KAM faible et théorie de Mather sur les systèmes lagrangiens, C. R. Acad. Sci. Paris Sér. I Math. 324 (1997), no. 9, 1043-1046.

[12] E. Garibaldi and A. O. Lopes, On the Aubry-Mather theory for symbolic dynamics, Ergodic Theory Dynam. Systems 28 (2008), no. 3, 791-815. 
[13] E. Garibaldi, A. O. Lopes, and Ph. Thieullen, On calibrated and separating sub-actions, Bull. Braz. Math. Soc. (N.S.) 40 (2009), no. 4, 577-602.

[14] Kazimierz Goebel and W. A. Kirk, Topics in metric fixed point theory, Cambridge University Press, Berlin, 1990.

[15] Oliver Jenkinson, Ergodic optimization, Discrete Contin. Dyn. Syst. 15 (2006), no. 1, 197-224.

[16] Renaud Leplaideur, A dynamical proof for the convergence of Gibbs measures at temperature zero, Nonlinearity 18 (2005), no. 6, 2847-2880.

[17] Ricardo Mañé, Generic properties and problems of minimizing measures of Lagrangian systems, Nonlinearity 9 (1996), no. 2, 273-310.

[18] Ian D Morris, Maximizing measures of generic hölder functions have zero entropy, Nonlinearity 21 (2008), 993-1000.

[19] Anthony Quas and Jason Siefken, Ergodic optimization of super-continuous functions on shift spaces, Ergodic Theory and Dynamical Systems 32 (2012), no. 6, 2071-2082.

[20] David Ruelle, Thermodynamic formalism. The mathematical structures of equilibrium statistical mechanics. 2nd edition., Cambridge Mathematical Library. Cambridge: Cambridge University Press, 2004.

[21] A. C. D. van Enter and W. M. Ruszel, Chaotic temperature dependence at zero temperature, J. Stat. Phys. 127 (2007), no. 3, 567-573.

[22] Peter Walters, An introduction to ergodic theory, Graduate Texts in Math. 79, Springer, 1982.

[23] G.C. Yuan and B.R. Hunt, Optimal orbits of hyperbolic systems, Nonlinearity 12 (1999), no. 4, 12071224 .

Cimat, A.P. 402, 36.000, Guanajuato. GTO, MÉxico.

E-mail address: gonzalo@cimat.mx 